


\title{
ADJOINT BASED A POSTERIORI ANALYSIS OF MULTISCALE MORTAR DISCRETIZATIONS WITH MULTINUMERICS
}

\author{
SIMON TAVENER* TIM WILDEY ${ }^{\dagger}$
}

\begin{abstract}
In this paper we derive a posteriori error estimates for linear functionals of the solution to an elliptic problem discretized using a multiscale nonoverlapping domain decomposition method. The error estimates are based on the solution of an appropriately defined adjoint problem. We present a general framework that allows us to consider both primal and mixed formulations of the forward and adjoint problems within each subdomain. The primal subdomains are discretized using either an interior penalty discontinuous Galerkin method or a continuous Galerkin method with weakly imposed Dirichlet conditions. The mixed subdomains are discretized using RaviartThomas mixed finite elements. The a posteriori error estimate also accounts for the errors due to adjoint-inconsistent subdomain discretizations. The coupling between the subdomain discretizations is achieved via a mortar space. We show that the numerical discretization error can be broken down into subdomain and mortar components which may be used to drive adaptive refinement.
\end{abstract}

Key words. mortar methods, multiscale, multinumerics, adjoint operators, error analysis, mixed finite elements, discontinuous Galerkin methods

AMS subject classifications. 65N55, 65N30, 65F08, 65F 10

1. Introduction. There has been considerable interest in recent years in solving multiphysics problems coupled through an interface. For example, geological reservoir models of flow in porous media often consist of multiple fault blocks which render a monolithic discretization infeasible $[4,61]$. In such instances it is desirable to be able to discretize each subdomain (fault block) independently and produce a numerical approximation using whichever technique is most appropriate. Finite volume methods are often preferred due to their local conservation properties and ease of implementation $[10,7,5,27]$, but these methods suffer in terms of accuracy in cases where highly distorted grids are required $[1,54]$. Alternatively, one may employ multipoint flux approximation methods $[1,3,54,55]$ or discontinuous Galerkin methods $[8,47,48,37,36]$, but these tend to be more expensive and should only be used where needed.

Mortar methods provide a convenient and mathematically elegant approach for coupling different numerical methods through physically meaningful interface conditions. They do not require the grids to match along the interface and can easily be generalized to multiphase flow [62, 43], multiphysics [61, 14], computational mechanics [33], and geomechanics [34]. The mortar mixed finite element method has also been shown to be equivalent to a multiscale method in the case where the mortar discretization is coarser than the subdomain discretizations [6]. This observation led to the development of a multiscale mortar basis implementation [31] as well as multiscale mortar preconditioners for stochastic mixed finite elements [53] and nonlinear interface operators [30]. Mortar methods have also been developed for interior penalty discontinuous Galerkin (DG) methods and the coupling of mixed and DG methods [35].

${ }^{*}$ Department of Mathematics, Colorado State University, 105 Weber Building, Ft. Collins, CO 80523, USA, (tavener@math.colostate.edu), phone: +1 970491 6452, fax: +1 9704912161

${ }^{\dagger}$ Sandia National Labs, Albuquerque, NM 87185. Sandia National Laboratories is a multiprogram laboratory managed and operated by Sandia Corporation, a wholly owned subsidiary of Lockheed Martin Corporation, for the U.S. Department of Energy's National Nuclear Security Administration under contract DE-AC04-94AL85000. (tmwilde@sandia.gov). 
In this paper, we allow each subdomain problem to be posed in either a primal or a mixed form. We present a general framework that allows each subdomain to be discretized using whichever method is most appropriate. We consider mixed finite elements, discontinuous Galerkin methods, and a continuous Galerkin method with weakly imposed Dirichlet conditions, but other methods, such as finite volumes, also fit within the general framework. Sufficient conditions for well-posedness of the multiscale mortar formulation has been previously established for all of the methods under consideration as well as the case of multinumerics (see e.g. $[6,35]$ ), which allows us to focus on the a posteriori error analysis.

Our objective is produce accurate a posteriori error estimates for certain quantities of interest (linear functionals) associated with the subdomain or mortar solutions. We use the solution of a global adjoint problem to define a dual-weighted residual that relates the error in the quantity of interest to a weak residual. This approach is well-established for steady-state problems [11, 32, 39], systems of ordinary differential equations [23, 24], time-dependent nonlinear systems [9, 26], multiphysics problems $[38,29]$, and problems solved via operator decomposition or operator splitting [28, $16,25]$. In this paper, we derive a general a posteriori error estimate for multiscale mortar discretizations that accounts for error due to both the subdomain and the mortar discretizations. We show that our approach can be used to estimate the error in linear functionals using any of the previously mentioned subdomain discretization techniques. We do not explicitly consider finite volume methods in this paper, but under certain circumstances the cell-centered finite volume scheme is equivalent to a mixed finite element method with an appropriate choice of quadrature [7,5]. Adjointbased a posteriori error estimates have been obtained by taking advantage of this equivalence in [27]. An extension of the a posteriori analysis in this paper to include the effect of quadrature can be pursued following [27]. Our error estimate decomposes the error into subdomain and mortar contributions which allows each discretization to be refined independently (subject to the usual mortar compatibility conditions). The error estimate also includes an adjoint inconsistency term which is important for some of the interior penalty discontinuous Galerkin methods [57].

A posteriori error estimates for mortar methods have appeared in $[60,59,12$, $13,42,44,56]$, but most of these estimate the error in the energy norm rather than the error in a quantity of interest. Many of these approaches involve bounds with unknown constants that must be estimated or bounded in order to compute the error estimate. One notable exception is [41] which is based on [21, 22, 51, 52] and explores the nontrivial extension of error estimates using the Prager-Synge inequality [45] to the case of multiscale mortar methods with multinumerics. A relatively recent paper [50] takes a similar approach to ours, but they consider a mortar formulation based on Neumann to Dirichlet maps (ours uses Dirichlet to Neumann maps) and does not consider multinumerics or multiscale mortar methods.

The remainder of this paper is organized as follows. In section 2, we introduce the model elliptic problem and decompose the problem into nonoverlapping subdomains in which either a primal or a mixed formulation is applied. In section 3, we define the adjoint to the model problem and provide the corresponding domain decomposition. In section 4, we define an abstract variational formulation of the forward and adjoint problems and we show how to reduce the problems to coarse scale interface operators. We derive an error representation for the abstract problem in section 5 and describe our adaptive mesh refinement strategy based on this error estimate in section 6 . In section 7 , we present some notation for specific variational formulations and we show 
that the general framework can be used to analyze the mixed finite element method, the interior penalty discontinuous Galerkin method, and the continuous Galerkin method with weakly imposed Dirichlet boundary conditions. Numerical results are presented in section 8 and our concluding remarks are in section 9.

2. Forward Problem. Let $\Omega \subset \mathbb{R}^{d}, d=2,3$, be a polygonal convex domain, and consider the model elliptic problem,

$$
\begin{cases}-\nabla \cdot(\mathbf{K} \nabla p)=f, & \text { in } \Omega, \\ p=g_{D}, & \text { on } \partial \Omega,\end{cases}
$$

where $\mathbf{K}$ is a symmetric, bounded, and uniformly positive definite tensor. We assume that $f$ and $g_{D}$ are sufficiently smooth so that the solution to (2.1) has the required regularity. We focus on Dirichlet bondary conditions for the sake of simplicity, but both Neumann and Robin boundary conditions can also be analyzed.

We decompose $\bar{\Omega}=\cup_{i=1}^{N} \bar{\Omega}_{i}$, which can be nonconforming in the sense that neighboring subdomains need not share complete sides (edges if $d=2$, faces if $d=3$ ). The subdomains are nonoverlapping, i.e., $\Omega_{i} \cap \Omega_{j}=\emptyset$ for $i \neq j$. We denote $\Gamma_{i, j}:=\partial \Omega_{i} \cap \partial \Omega_{j}, \Gamma:=\cup_{1 \leq i<j \leq n} \Gamma_{i, j}$, and $\Gamma_{i}:=\partial \Omega_{i} \cap \Gamma$. We define the index sets $I_{P}$ and $I_{M}$ where for $i \in I_{P}$ we consider the primal formulation

$$
\begin{cases}-\nabla \cdot\left(\mathbf{K} \nabla p_{i}\right)=f, & \text { in } \Omega_{i}, \\ p_{i}=g_{D}, & \text { on } \partial \Omega_{i} \cap \partial \Omega, \\ p_{i}=\lambda, & \text { on } \Gamma_{i},\end{cases}
$$

and $i \in I_{M}$ we consider the mixed formulation,

$$
\begin{cases}\mathbf{u}_{i}=-\mathbf{K} \nabla p_{i}, & \text { in } \Omega_{i}, \\ \nabla \cdot \mathbf{u}_{i}=f, & \text { in } \Omega_{i}, \\ p_{i}=g_{D}, & \text { on } \partial \Omega_{i} \cap \partial \Omega, \\ p_{i}=\lambda, & \text { on } \Gamma_{i} .\end{cases}
$$

The model problem is closed by imposing continuity of the normal flux,

$$
\begin{cases}-\mathbf{u}_{i} \cdot \mathbf{n}_{i}=\mathbf{u}_{j} \cdot \mathbf{n}_{j}, & i, j \in I_{M}, \\ \mathbf{u}_{i} \cdot \mathbf{n}_{i}=\mathbf{K} \nabla p_{j} \cdot \mathbf{n}_{j}, & i \in I_{M}, j \in I_{P}, \\ \mathbf{K} \nabla p_{i} \cdot \mathbf{n}_{i}=-\mathbf{K} \nabla p_{j} \cdot \mathbf{n}_{j}, & i, j \in I_{P}\end{cases}
$$

for all $i, j$ such that $\partial \Omega_{i} \cap \partial \Omega_{j} \neq \emptyset$, where $\mathbf{n}_{i}$ is the outwards facing normal vector to $\partial \Omega_{i}$. It is well known (see e.g., $\left.[46,35]\right)$ that $(2.1)$ and $(2.2)-(2.4)$ are equivalent if we set $\lambda=\left.p\right|_{\Gamma}$.

3. Adjoint Problem. The formal adjoint for the model problem is,

$$
\begin{cases}-\nabla \cdot(\mathbf{K} \nabla \xi)=\psi, & \text { in } \Omega, \\ \xi=0, & \text { on } \partial \Omega_{i} \cap \partial \Omega,\end{cases}
$$

where $\psi$ is chosen based on the quantity of interest. We remark that inhomogeneous boundary conditions can be chosen for the adjoint if the quantity of interest is on the boundary (see e.g., [58]). The boundary conditions on the adjoint problem depend 
on the boundary conditions for the forward problem and must be defined properly if the forward problem has Neumann or Robin conditions. This issue is well-known, so we do not address it further in this paper.

For $i \in I_{P}$ we consider the primal formulation of the adjoint,

$$
\begin{cases}-\nabla \cdot\left(\mathbf{K} \nabla \xi_{i}\right)=\psi, & \text { in } \Omega_{i}, \\ \xi_{i}=0, & \text { on } \partial \Omega_{i} \cap \partial \Omega, \\ \xi_{i}=\eta, & \text { on } \Gamma_{i},\end{cases}
$$

and $i \in I_{M}$ we consider the mixed formulation of the adjoint,

$$
\begin{cases}\mathbf{s}_{i}=\mathbf{K} \nabla \xi_{i}, & \text { in } \Omega_{i}, \\ -\nabla \cdot \mathbf{s}_{i}=\psi, & \text { in } \Omega_{i}, \\ \xi_{i}=0, & \text { on } \partial \Omega_{i} \cap \partial \Omega \\ \xi_{i}=\eta, & \text { on } \Gamma_{i} .\end{cases}
$$

The adjoint problem is closed by imposing continuity of the normal flux,

$$
\begin{cases}\mathbf{s}_{i} \cdot \mathbf{n}_{i}=-\mathbf{s}_{j} \cdot \mathbf{n}_{j}, & i, j \in I_{M}, \\ \mathbf{s}_{i} \cdot \mathbf{n}_{i}=-\mathbf{K} \nabla \xi_{j} \cdot \mathbf{n}_{j}, & i \in I_{M}, j \in I_{P}, \\ \mathbf{K} \nabla \xi_{i} \cdot \mathbf{n}_{i}=-\mathbf{K} \nabla \xi_{j} \cdot \mathbf{n}_{j}, & i, j \in I_{P}\end{cases}
$$

for all $i, j$ such that $\partial \Omega_{i} \cap \partial \Omega_{j} \neq \emptyset$. It is clear that (3.1) and (3.2)-(3.4) are equivalent if we set $\eta=\left.\xi\right|_{\Gamma}$. Note that we have defined the adjoint velocity, $\mathbf{s}_{i}$ to be $\mathbf{K} \nabla \xi_{i}$ rather than $-\mathbf{K} \nabla \xi_{i}$. This choice makes (3.3) the adjoint of the forward mixed formulation (2.3).

Our quantity of interest may be a linear functional of $\lambda$ on $\Gamma$. In this case, we write the quantity of interest as,

$$
j_{M}(\lambda)=\left\langle\psi_{M}, \lambda\right\rangle_{\Gamma}
$$

and we modify the adjoint interface condition (3.4) to,

$$
\begin{cases}\mathbf{s}_{i} \cdot \mathbf{n}_{i}=-\mathbf{s}_{j} \cdot \mathbf{n}_{j}+\psi_{M}, & i, j \in I_{M}, \\ \mathbf{s}_{i} \cdot \mathbf{n}_{i}=-\mathbf{K} \nabla \xi_{j} \cdot \mathbf{n}_{j}+\psi_{M}, & i \in I_{M}, j \in I_{P}, \\ \mathbf{K} \nabla \xi_{i} \cdot \mathbf{n}_{i}=-\mathbf{K} \nabla \xi_{j} \cdot \mathbf{n}_{j}+\psi_{M}, & i, j \in I_{P} .\end{cases}
$$

4. Abstract Variational Formulations. In this section, we define the variational formulations associated with (2.2)-(2.4) and the discretized variants. To simplify the discussion, we first define a consistent notation to be used throughout the paper. Then, following $[6,35]$, we show how to reduce the global problem to an interface problem, which corresponds to a multiscale finite element method if a coarse scale discrete mortar space is chosen.

4.1. Variational Formulation of the Forward Problem. On a subdomain, $\Omega_{i}$, we let $\mathbf{V}_{i}$ and $M$ denote abstract Hilbert spaces and let $\mathcal{A}_{i}: \mathbf{V}_{i} \times \mathbf{V}_{i} \rightarrow \mathbb{R}$ and $\mathcal{B}_{i}: M \times \mathbf{V}_{i} \rightarrow \mathbb{R}$ denote appropriate continuous bilinear forms. We define a variational formulation seeking $\mathbf{z}_{i} \in \mathbf{V}_{i}$ such that,

$$
\mathcal{A}_{i}\left(\mathbf{z}_{i}, \mathbf{w}_{i}\right)=l_{i}\left(\mathbf{w}_{i}\right)-\mathcal{B}_{i}\left(\lambda, \mathbf{w}_{i}\right), \quad \forall \mathbf{w}_{i} \in \mathbf{V}_{i}
$$


where $l_{i}: \mathbf{V}_{i} \rightarrow \mathbb{R}$ is a continuous linear form. We assume that each variational formulation is consistent in the sense that it is equivalent to either (2.2) or (2.3). Examples of consistent variational formulations will be provided in subsequent sections.

In order to impose the interface condition (2.4), we require two additional continuous bilinear forms: $\mathcal{C}_{i}: \mathbf{V}_{i} \times M \rightarrow \mathbb{R}$ and $\mathcal{D}_{i}: M \times M \rightarrow \mathbb{R}$. The global problem seeks $\mathbf{z}_{i} \in \mathbf{V}_{i}$ and $\lambda \in M$ such that

$$
\mathcal{A}_{i}\left(\mathbf{z}_{i}, \mathbf{w}_{i}\right)=l_{i}\left(\mathbf{w}_{i}\right)-\mathcal{B}_{i}\left(\lambda, \mathbf{w}_{i}\right), \quad \forall \mathbf{w}_{i} \in \mathbf{V}_{i},
$$

for $i=1, \ldots, N$ and

$$
\sum_{i=1}^{N}\left(\mathcal{C}_{i}\left(\mathbf{z}_{i}, \mu\right)+\mathcal{D}_{i}(\lambda, \mu)\right)=0, \quad \forall \mu \in M .
$$

Note that $\mathbf{z}_{i}$ is defined locally and $\lambda$ is defined globally. Examples of these bilinear forms will also be provided in subsequent sections.

REMARK 4.1. We assume that the variational formulation is consistent, which implies that

$$
\mathcal{C}_{i}\left(\mathbf{z}_{i}, \mu\right)+\mathcal{D}_{i}(\lambda, \mu)= \begin{cases}-\left\langle\mathbf{u}_{i} \cdot \mathbf{n}_{i}, \mu\right\rangle_{\Gamma_{i}}, & i \in I_{M}, \\ \left\langle\mathbf{K} \nabla p_{i} \cdot \mathbf{n}_{i}, \mu\right\rangle_{\Gamma_{i}}, & i \in I_{P},\end{cases}
$$

but this does not necessarily require that $\mathcal{D}_{i}(\lambda, \mu)=0$. Since $\lambda=\left.p\right|_{\Gamma}$, we may allow $\mathcal{D}_{i}(\lambda, \mu)$ to be nonzero and modify $\mathcal{C}_{i}\left(\mathbf{z}_{i}, \mu\right)$ appropriately to maintain consistency. For example, in Section 7.2 we use penalization to weakly enforce the Dirichlet boundary conditions in a consistent manner.

On each subdomain, the exact solution is unknown so we choose a discrete subspace $\mathbf{V}_{h, i} \subset \mathbf{V}_{i}$ associated with the partition $\mathcal{T}_{h, i}$ and seek $\mathbf{z}_{h, i} \in \mathbf{V}_{h, i}$ such that,

$$
\mathcal{A}_{i}\left(\mathbf{z}_{h, i}, \mathbf{w}_{i}\right)=l_{i}\left(\mathbf{w}_{i}\right)-\mathcal{B}_{i}\left(\lambda, \mathbf{w}_{i}\right), \quad \forall \mathbf{w}_{i} \in \mathbf{V}_{h, i} .
$$

Similarly, the boundary condition on the interface is unknown, so we choose a discrete subspace $M_{H} \subset M$. The discrete global problem seeks $\mathbf{z}_{h, i} \in \mathbf{V}_{h, i}$ and $\lambda_{H} \in M_{H}$ such that

$$
\mathcal{A}_{i}\left(\mathbf{z}_{h, i}, \mathbf{w}_{i}\right)=l_{i}\left(\mathbf{w}_{i}\right)-\mathcal{B}_{i}\left(\lambda_{H}, \mathbf{w}_{i}\right), \quad \forall \mathbf{w}_{i} \in \mathbf{V}_{h, i},
$$

for $i=1, \ldots, N$ and

$$
\sum_{i=1}^{N}\left(\mathcal{C}_{i}\left(\mathbf{z}_{h, i}, \mu\right)+\mathcal{D}_{i}\left(\lambda_{H}, \mu\right)\right)=0, \quad \forall \mu \in M_{H} .
$$

Even if the continuous global problem (4.2) - (4.3) and the discrete local problem (4.4) are well-posed, the discrete global problem (4.5) - (4.6) may not be. A compatibility condition may exist between $\mathbf{V}_{h, i}$ and $M_{H}$. This issue has been studied extensively for mortar mixed finite elements (see e.g., $[4,6]$ ) and the general conclusion is that the mortar space cannot be too rich compared with the trace of the subdomain discretization. In this paper, we are mostly interested in coarse scale mortar discretizations so the compatibility condition is easily satisfied and we will not address this issue further.

EXAMPLE 4.2. In the case of two subdomains, the global discrete problem has the form:

$$
\left[\begin{array}{ccc}
A_{1} & & B_{1} \\
& A_{2} & B_{2} \\
C_{1} & C_{2} & \left(D_{1}+D_{2}\right)
\end{array}\right]\left[\begin{array}{c}
z_{1} \\
z_{2} \\
\lambda
\end{array}\right]=\left[\begin{array}{c}
l_{1} \\
l_{2} \\
0
\end{array}\right]
$$


where, e.g., $A_{i}$ is the matrix corresponding to $\mathcal{A}_{i}(\cdot, \cdot)$ and $l_{i}$ is the vector corresponding to $l_{i}(\cdot)$.

Let $\mathbf{e}_{z, i}=\mathbf{z}_{i}-\mathbf{z}_{h, i}$ and $e_{\lambda}=\lambda-\lambda_{H}$ denote the error in the subdomain and mortar approximations which satisfy the following orthogonality relations. Subtracting (4.5) from (4.2) and (4.6) from (4.3),

$$
\mathcal{A}_{i}\left(\mathbf{e}_{z, i}, \mathbf{w}_{i}\right)=-\mathcal{B}_{i}\left(e_{\lambda}, \mathbf{w}_{i}\right), \quad \forall \mathbf{w}_{i} \in \mathbf{V}_{h, i}
$$

for $i=1, \ldots, N$ and

$$
\sum_{i=1}^{N}\left(\mathcal{C}_{i}\left(\mathbf{e}_{z, i}, \mu\right)+\mathcal{D}_{i}\left(e_{\lambda}, \mu\right)\right)=0, \quad \forall \mu \in M_{H} .
$$

Finally, we let $\boldsymbol{\Pi}_{h, i}: \mathbf{V}_{i} \rightarrow \mathbf{V}_{h, i}$ and $\mathcal{Q}_{H}: M \rightarrow M_{H}$ denote projection operators into the discrete subspaces. Examples of $\boldsymbol{\Pi}_{h, i}$ and $\mathcal{Q}_{H}$ will be provided in subsequent sections.

4.2. Abstract Variational Formulation of the Adjoint. On each subdomain, $\Omega_{i}$ we let $\mathcal{A}_{i}^{*}: \mathbf{V}_{i} \times \mathbf{V}_{i} \rightarrow \mathbb{R}, \mathcal{C}_{i}^{*}: M \times \mathbf{V}_{i} \rightarrow \mathbb{R}, \mathcal{B}_{i}^{*}: \mathbf{V}_{i} \times M \rightarrow \mathbb{R}$, and $\mathcal{D}_{i}^{*}: M \times M \rightarrow \mathbb{R}$. denote appropriate continuous adjoint bilinear forms. The global problem seeks $\phi_{i} \in \mathbf{V}_{i}$ and $\eta \in M$ such that

$$
\mathcal{A}_{i}^{*}\left(\phi_{i}, \mathbf{w}_{i}\right)=j_{i}\left(\mathbf{w}_{i}\right)-\mathcal{C}_{i}^{*}\left(\eta, \mathbf{w}_{i}\right), \quad \forall \mathbf{w}_{i} \in \mathbf{V}_{i},
$$

for $i=1, \ldots, N$ and

$$
\sum_{i=1}^{N}\left(\mathcal{B}_{i}^{*}\left(\phi_{i}, \mu\right)+\mathcal{D}_{i}^{*}(\eta, \mu)\right)=j_{M}(\mu), \quad \forall \mu \in M,
$$

where $j_{i}: \mathbf{V}_{i} \rightarrow \mathbb{R}$ and $j_{M}: M \rightarrow \mathbb{R}$ are continuous linear forms associated with subdomain and mortar quantities of interest respectively. We assume that this adjoint variational formulation is consistent in the sense that it is equivalent to (3.1). Examples of adjoint linear and bilinear forms will be provided in subsequent sections.

EXAMPLE 4.3. In the case of two subdomains, the global discrete adjoint problem has the form:

$$
\left[\begin{array}{ccc}
A_{1}^{*} & & C_{1}^{*} \\
& A_{2}^{*} & C_{2}^{*} \\
B_{1}^{*} & B_{2}^{*} & \left(D_{1}^{*}+D_{2}^{*}\right)
\end{array}\right]\left[\begin{array}{c}
\phi_{1} \\
\phi_{2} \\
\eta
\end{array}\right]=\left[\begin{array}{c}
j_{1} \\
j_{2} \\
j_{M}
\end{array}\right]
$$

where, e.g., $A_{i}^{*}$ is the matrix corresponding to $\mathcal{A}_{i}^{*}(\cdot, \cdot)$ and $j_{i}$ is the vector corresponding to $j_{i}(\cdot)$.

We emphasize that the linear and bilinear forms in (4.9)-(4.10) are derived from either the primal form or the mixed form of the formal adjoint. We do not assume that the variational forms used for the forward problem are adjoint consistent, which would imply that they are also variational forms for the adjoint. To be precise, we do not assume

$$
\mathcal{A}_{i}\left(\mathbf{z}_{i}, \mathbf{w}_{i}\right)=\mathcal{A}_{i}^{*}\left(\mathbf{w}_{i}, \mathbf{z}_{, i}\right), \quad \forall \mathbf{w}_{i}, \mathbf{z}_{i} \in \mathbf{V}_{i},
$$

nor do we necessarily assume

$$
\mathcal{B}_{i}\left(\lambda, \mathbf{w}_{i}\right)=\mathcal{B}_{i}^{*}\left(\mathbf{w}_{i}, \lambda\right), \quad \mathcal{C}_{i}\left(\mathbf{z}_{i}, \mu\right)=\mathcal{C}_{i}^{*}\left(\mu, \mathbf{z}_{i}\right), \quad \mathcal{D}_{i}(\lambda, \mu)=\mathcal{D}_{i}^{*}(\mu, \lambda),
$$


for all $\lambda, \mu \in M$ and $\mathbf{w}_{i}, \mathbf{z}_{i} \in \mathbf{V}_{i}$. The issue of adjoint consistency will be important in subsequent sections since the adjoint inconsistent variational formulations require additional terms in the error representation. As it turns out, all of the forward and adjoint bilinear forms considered in this paper satisfy (4.12), but the error analysis in Section 5 does not make this assumption.

4.3. Reduction to Interface Problems. In the multiscale setting, the number of degrees of freedom associated with the mortar space may be relatively small in comparison with the subdomain degrees of freedom. In this case, it may be more economical to construct the coarse scale interface operator (a.k.a Steklov-Poincare operator or Schur complement [46]) and solve the interface problem directly rather than using a standard iterative nonoverlapping domain decomposition approach which requires subdomain solves at each iteration.

Following [4, 6], we define a bilinear form $d_{H}: L^{2}(\Gamma) \times L^{2}(\Gamma) \rightarrow \mathbb{R}$ by

$$
d_{H}(\lambda, \mu)=\sum_{i=1}^{N}\left(\mathcal{D}_{i}(\lambda, \mu)+\mathcal{C}_{i}\left(\hat{\mathbf{z}}_{h, i}(\lambda), \mu\right)\right)
$$

where $\hat{\mathbf{z}}_{h, i}(\lambda) \in \mathbf{V}_{h, i}$ satisfies

$$
\mathcal{A}_{i}\left(\hat{\mathbf{z}}_{h, i}(\lambda), \mathbf{w}_{i}\right)=-\mathcal{B}_{i}\left(\lambda, \mathbf{w}_{i}\right), \quad \forall \mathbf{w}_{i} \in \mathbf{V}_{h, i} .
$$

We define a linear form $g_{H}: L^{2}(\Gamma) \rightarrow \mathbb{R}$ by

$$
g_{H}(\mu)=-\sum_{i=1}^{N} \mathcal{C}_{i}\left(\overline{\mathbf{z}}_{h, i}, \mu\right),
$$

where $\overline{\mathbf{z}}_{h, i} \in \mathbf{V}_{h, i}$ satisfies

$$
\mathcal{A}_{i}\left(\overline{\mathbf{z}}_{h, i}, \mathbf{w}_{i}\right)=l_{i}\left(\mathbf{w}_{i}\right), \quad \forall \mathbf{w}_{i} \in \mathbf{V}_{h, i} .
$$

It is easy to verify that the solution of (4.5) - (4.6) also satisfies,

$$
d_{H}\left(\lambda_{H}, \mu\right)=g_{H}(\mu), \quad \forall \mu \in M_{H}
$$

and that

$$
\mathbf{z}_{h, i}=\hat{\mathbf{z}}_{h, i}(\lambda)+\overline{\mathbf{z}}_{h, i} .
$$

EXAMPLE 4.4. In the case of two subdomains, the discrete interface problem has the form:

$$
\left(\left(D_{1}-C_{1} A_{1}^{-1} B_{1}\right)+\left(D_{2}-C_{2} A_{2}^{-1} B_{2}\right)\right) \lambda=-C_{1} A_{1}^{-1} l_{1}-C_{2} A_{2}^{-1} l_{2},
$$

which is clearly recognized as the Schur complement of the subdomain matrices. The the left-hand and right-hand sides are the discrete representations of (4.13) and (4.15) respectively.

We use (4.14) and (4.16) write the interface operator as,

$d_{H}\left(\lambda_{H}, \mu\right)=\sum_{i=1}^{N}\left(\mathcal{A}_{i}\left(\hat{\mathbf{z}}_{h, i}\left(\lambda_{H}\right), \hat{\mathbf{z}}_{h, i}(\mu)\right)+\mathcal{B}_{i}\left(\lambda_{H}, \hat{\mathbf{z}}_{h, i}(\mu)\right)+\mathcal{C}_{i}\left(\hat{\mathbf{z}}_{h, i}\left(\lambda_{H}\right), \mu\right)+\mathcal{D}_{i}\left(\lambda_{H}, \mu\right)\right)$, 
for all $\lambda_{H}, \mu \in M_{H}$. We remark that (4.18) is a generalization of the characterization given in [6] for mortar mixed finite elements. Given (4.18), we can easily show that symmetry and positive definiteness of the interface operator follow from the properties of the subdomain bilinear forms and the aforementioned compatibility condition between the discrete mortar space and the discrete subdomain spaces.

We can follow the same procedure and reduce the adjoint problem (4.9)-(4.10) to an interface problem of the form,

$$
d_{H}^{*}(\eta, \mu)=g_{H}^{*}(\mu) .
$$

We omit the details for the sake of brevity, but we remark that the interface operator, $d_{H}^{*}(\cdot, \cdot)$, does not depend on the adjoint data, i.e., the quantity of interest. Therefore, we can investigate multiple quantities of interest and we only need to compute multiple right-hand sides for (4.19) which only involves one or two additional solves per subdomain depending on the implementation. Furthermore, many quantities of interest are local and we only need to solve additional subdomain problems where the adjoint data is nonzero.

5. Error Analysis. In this section, we use the global adjoint formulation (4.9) - (4.10) to derive an a posteriori error estimate for discrete approximations of the global forward problem (4.2) - (4.3). Our analysis is based on the generic linear and bilinear forms introduced in the previous section, and specific examples will be given in the next section.

For $1 \leq i \leq N$, let $\mathbf{z}_{i} \in \mathbf{V}_{i}$ and $\lambda \in M$ solve (4.2) - (4.3), and let $\mathbf{z}_{h, i} \in \mathbf{V}_{h, i}$ and $\lambda_{H} \in M_{H}$ solve (4.5) - (4.6). We recall that

$$
\mathbf{e}_{z, i}=\mathbf{z}_{i}-\mathbf{z}_{h, i}, \quad \text { and } \quad e_{\lambda}=\lambda-\lambda_{H},
$$

denote the subdomain and mortar errors respectively. Finally, for $1 \leq i \leq N$, let $\phi_{i} \in \mathbf{V}_{i}$ and $\eta \in M$ solve (4.9) - (4.10). We first present the theoretical result for the cases where (4.11) and (4.12) are satisfied.

Theorem 5.1. For $1 \leq i \leq N$, let $\mathbf{z}_{i} \in \mathbf{V}_{i}$ and $\lambda \in M$ solve (4.2) - (4.3), let $\mathbf{z}_{h, i} \in \mathbf{V}_{h, i}$ and $\lambda_{H} \in M_{H}$ solve (4.5) - (4.6), and let $\phi_{i} \in \mathbf{V}_{i}$ and $\eta \in M$ solve (4.9) - (4.10). Suppose (4.11) and (4.12) are satisfied. The error in a linear functional of the solution satisfies,

$$
j_{M}\left(e_{\lambda}\right)+\sum_{i=1}^{N} j_{i}\left(\mathbf{e}_{z, i}\right)=\sum_{i=1}^{N}\left(\mathcal{E}_{s u b, i}+\mathcal{E}_{\text {mort }, i}\right),
$$

where

$$
\mathcal{E}_{\text {sub }, i}=l_{i}\left(\phi_{i}-\Pi_{h, i} \phi_{i}\right)-\mathcal{A}_{i}\left(\mathbf{z}_{h, i}, \phi_{i}-\Pi_{h, i} \phi_{i}\right)-\mathcal{B}_{i}\left(\lambda_{H}, \phi_{i}-\Pi_{h, i} \phi_{i}\right),
$$

represents the subdomain discretization error, and

$$
\mathcal{E}_{\text {mort }, i}=-\mathcal{C}_{i}\left(\mathbf{z}_{h, i}, \eta-\mathcal{Q}_{H} \eta\right)-\mathcal{D}_{i}\left(\lambda_{H}, \eta-\mathcal{Q}_{H} \eta\right),
$$

represents the contribution to the mortar discretization error.

Proof. We set $\mathbf{w}_{i}=\mathbf{e}_{z, i}$ in (4.9) and $\mu=e_{\lambda}$ in (4.10), giving

$$
\begin{aligned}
j_{M}\left(e_{\lambda}\right)+\sum_{i=1}^{N} j_{i}\left(\mathbf{e}_{z, i}\right) & =\sum_{i=1}^{N}\left(\mathcal{A}_{i}^{*}\left(\boldsymbol{\phi}_{i}, \mathbf{e}_{z, i}\right)+\mathcal{C}_{i}^{*}\left(\eta, \mathbf{e}_{z, i}\right)+\mathcal{B}_{i}^{*}\left(\boldsymbol{\phi}_{i}, e_{\lambda}\right)+\mathcal{D}_{i}^{*}\left(\eta, e_{\lambda}\right)\right) \\
& =\sum_{i=1}^{N}\left(\mathcal{A}_{i}\left(\mathbf{e}_{z, i}, \boldsymbol{\phi}_{i}\right)+\mathcal{B}_{i}\left(e_{\lambda}, \boldsymbol{\phi}_{i}\right)+\mathcal{C}_{i}\left(\mathbf{e}_{z, i}, \eta\right)+\mathcal{D}_{i}\left(, e_{\lambda}, \eta\right)\right),
\end{aligned}
$$


where we have utilized (4.11) and (4.12). The remainder of the proof follows the usual steps utilizing Galerkin orthogonality (4.7)-(4.8) with the projection operators defined in section 4 , and the definition of the weak residual.

Unfortunately, some bilinear forms may not satisfy (4.11) or (4.12). To account for this possibility, we introduce the adjoint inconsistency term,

$$
\begin{aligned}
\mathcal{E}_{\mathrm{cons}, i}=\mathcal{A}_{i}^{*}\left(\phi_{i}, \mathbf{e}_{z, i}\right)-\mathcal{A}_{i}\left(\mathbf{e}_{z, i}, \phi_{i}\right)+\mathcal{C}_{i}^{*}\left(\eta, \mathbf{e}_{z, i}\right)-\mathcal{C}_{i}\left(\mathbf{e}_{z, i}, \eta\right) & \\
& +\mathcal{B}_{i}^{*}\left(\phi_{i}, e_{\lambda}\right)-\mathcal{B}_{i}\left(e_{\lambda}, \phi_{i}\right)+\mathcal{D}_{i}^{*}\left(\eta, e_{\lambda}\right)-\mathcal{D}_{i}\left(e_{\lambda}, \eta\right)
\end{aligned}
$$

This term must be estimated (or bounded) in order to derive a fully computable error representation. In Section 7.2, we provide an example where (4.11) is not satisfied and $\mathcal{E}_{\text {cons }, i}$ is computable.

The following result can be shown using a straightforward modification of the proof of Theorem 5.1.

Corollary 5.2. For $1 \leq i \leq N$, let $\mathbf{z}_{i} \in \mathbf{V}_{i}$ and $\lambda \in M$ solve (4.2) - (4.3), let $\mathbf{z}_{h, i} \in \mathbf{V}_{h, i}$ and $\lambda_{H} \in M_{H}$ solve (4.5) - (4.6), and let $\phi_{i} \in \mathbf{V}_{i}$ and $\eta \in M$ solve (4.9) - (4.10). Suppose (4.11) and (4.12) are not satisfied. The error in a linear functional of the solution satisfies,

$$
j_{M}\left(e_{\lambda}\right)+\sum_{i=1}^{N} j_{i}\left(\mathbf{e}_{z, i}\right)=\sum_{i=1}^{N}\left(\mathcal{E}_{\text {sub }, i}+\mathcal{E}_{\text {mort }, i}+\mathcal{E}_{\text {cons }, i}\right),
$$

where $\mathcal{E}_{\text {cons }, i}$ represents the consistency error given by $(5.2), \mathcal{E}_{\text {sub }, i}$ represents the subdomain discretization error, and $\mathcal{E}_{\text {mort }, i}$ represents the contribution to the mortar discretization error.

REMARK 5.3. A different error representation for adjoint inconsistent formulations can be derived using the adjoint the forward variational problem. While this approach does not require estimating the adjoint inconsistency terms, it has been found to be problematic for certain adjoint inconsistent formulations [37, 36]. In this paper, we follow the approach taken in [57] and use a variational formulation of the continuous adjoint and assume that the adjoint inconsistency terms can be estimated.

6. Adaptive Mesh Refinement. In this section, we decompose the error representation (5.1) into a sum of contributions from each element and use these localized error representations to drive adaptive mesh refinement. The standard approach, utilized in e.g. [11, 9, 20, 28, 16, 57], computes the absolute value of the localized error representation,

$$
\begin{aligned}
\mathcal{E}_{\mathrm{sub}, i, j} & =\left|l_{i, j}\left(\phi_{i}-\boldsymbol{\Pi}_{h, i} \phi_{i}\right)-\mathcal{A}_{i, j}\left(\mathbf{z}_{h, i}, \phi_{i}-\boldsymbol{\Pi}_{h, i} \phi_{i}\right)-\mathcal{B}_{i, j}\left(\lambda_{H}, \phi_{i}-\boldsymbol{\Pi}_{h, i} \phi_{i}\right)\right| \\
\mathcal{E}_{\mathrm{mort}, i, j} & =\left|-\mathcal{C}_{i, j}\left(\mathbf{z}_{h, i}, \eta-\mathcal{Q}_{H} \eta\right)-\mathcal{D}_{i, j}\left(\lambda_{H}, \eta-\mathcal{Q}_{H} \eta\right)\right|,
\end{aligned}
$$

where we have used the obvious notation to denote the localization of the linear and bilinear forms to a subdomain element $E_{j}$ or a mortar element $\gamma_{j}$. Next, we mark for refinement either a certain percentage of the elements or those where the local indicator exceeds a given tolerance. It is well known that this approach does not account for the cancellation of error between elements and is not guaranteed to reduce the error in the quantity of interest. Nevertheless, this approach has proven to be quite successful in practice and we consider a modification of this adaptive algorithm to allow adaptive refinement of both the mortar and the subdomain discretizations.

REMARK 6.1. For some discretization techniques, such as discontinuous Galerkin, the error representation (5.1) involves a sum of integrals over the edges (faces) in the 
mesh. In such cases, we allocate half of the edge contribution to both of the elements sharing the edge in order to localize the error to the elements for refinement. An alternative approach may be to use a hybridized discontinuous Galerkin method (see e.g., [19]) and to separately refine the element and edge discretization, but this is beyond the scope of this work.

We can also significantly reduce the overall computational cost by solving on each subdomain only a few times when the error is relatively large. We achieve this by starting with a relatively coarse mortar discretization and refining only the subdomains until the subdomain contribution is below a given tolerance. Then we refine the mortar discretization until the mortar contribution is below the tolerance. Since the subdomain meshes are refined first and remain fixed as the mortars are adapted, we can reuse certain subdomain information, such as the factorizations of the subdomain linear systems or the Krylov subspaces, for each iteration of mortar refinement.

We summarize our adaptive refinement algorithm in Alg. 1. Within the mortar

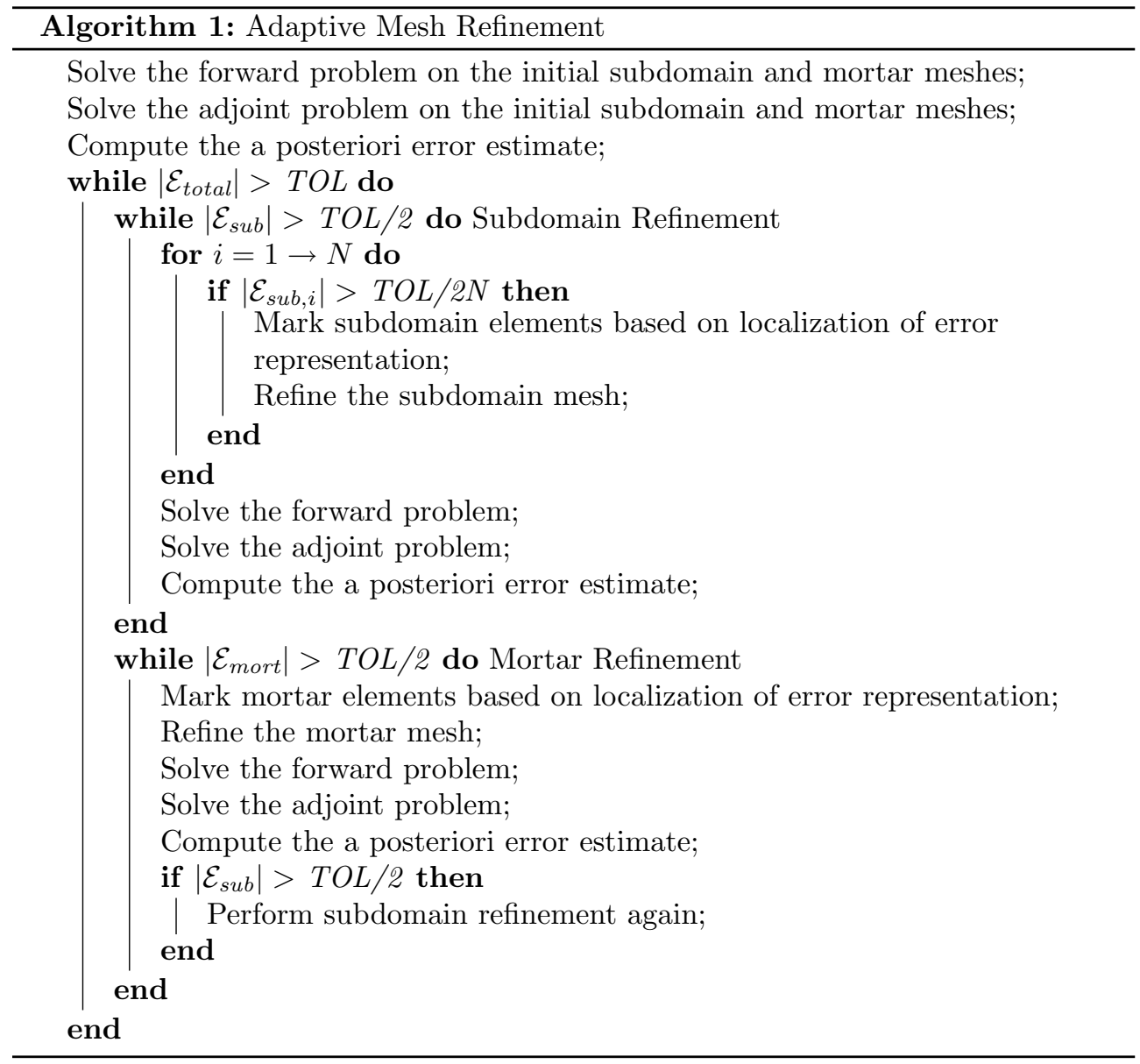

adaptive loop we account for the possibility that refining the mortars may affect the subdomain error estimates. This can occur if the error in the subdomain approximation is particularly sensitive to the boundary data. In most instances, while the 
subdomain solution clearly depends on the boundary data, the subdomain error is relatively insensitive to variations in the boundary data and this additional subdomain refinement is rarely required.

7. Examples of Discretized Variational Formulations. In a region $\Omega$, the scalar product of $L^{2}(\Omega)$ is denoted by $(\cdot, \cdot)_{\Omega}$

$$
\forall f, g \in L^{2}(\Omega),(f, g)_{\Omega}=\int_{\Omega} f(x) g(x) d x,
$$

and if the domain of integration is clear from the context, we suppress the index $\Omega$. For any non-negative integer $m$, recall the classical Sobolev space (cf. [2])

$$
H^{m}(\Omega)=\left\{v \in L^{2}(\Omega) ; \partial^{k} v \in L^{2}(\Omega) \forall|k| \leq m\right\},
$$

equipped with the following seminorm and norm

$$
|v|_{H^{m}(\Omega)}=\left[\sum_{|k|=m} \int_{\Omega}\left|\partial^{k} v\right|^{2} d x\right]^{1 / 2},\|v\|_{H^{m}(\Omega)}=\left[\sum_{0 \leq|k| \leq m}|v|_{H^{k}(\Omega)}^{2}\right]^{1 / 2} .
$$

This definition is extended to any real number $s=m+s^{\prime}$ for an integer $m \geq 0$ and $0<s^{\prime}<1$ by defining in dimension $d$ the fractional semi-norm and norm:

$$
\begin{gathered}
|v|_{H^{s}(\Omega)}=\left(\sum_{|k|=m} \int_{\Omega} \int_{\Omega} \frac{\left|\partial^{k} v(x)-\partial^{k} v(y)\right|^{2}}{|x-y|^{d+2 s^{\prime}}} d x d y\right)^{1 / 2} \\
\|v\|_{H^{s}(\Omega)}=\left(\|v\|_{H^{m}(\Omega)}^{2}+|v|_{H^{s}(\Omega)}^{2}\right)^{1 / 2}
\end{gathered}
$$

We also use the space of functions with finite divergence,

$$
\mathbf{H}(\operatorname{div}, \Omega)=\left\{\mathbf{v} \in \boldsymbol{L}^{2}(\Omega) ; \nabla \cdot \mathbf{v} \in L^{2}(\Omega)\right\} .
$$

Let $\mathcal{T}_{h, i}$ be a conforming partition of $\Omega_{i}$, consisting of simplices or parallelopipeds of maximum diameter $h_{i}$. We assume that the mesh is regular in the sense of Ciarlet [18], but we do not assume $\mathcal{T}_{h, i}$ and $\mathcal{T}_{h, j}$ align along $\Gamma_{i, j}$. We use $\mathcal{E}_{h, i}$ to denote the interior sides of $\mathcal{T}_{h, i}$, i.e., $\mathcal{E}_{h, i}$ does not contain the outer boundary of $\Omega_{i}$. We let $\mathcal{E}_{h, i}^{\text {ext }}$ be the partition of $\partial \Omega_{i} \cap \partial \Omega$ by the sides of $\mathcal{T}_{h, i}$. Similarly, we let $\mathcal{E}_{h, i}^{\Gamma}$ denote the partition of $\partial \Omega_{i} \cap \Gamma$ by the sides of $\mathcal{T}_{h, i}$.

We also require the broken Sobolev space associated with $\mathcal{T}_{h, i}$ given by,

$$
H^{m}\left(\mathcal{T}_{h, i}\right):=\left\{v \in L^{2}\left(\Omega_{i}\right):\left.v\right|_{T} \in H^{m}(T), \forall T \in \mathcal{T}_{h, i}\right\}, \quad m>3 / 2,
$$

equipped with the following norm,

$$
\|v\|_{m, \Omega_{i}}=\left(\sum_{T \in \mathcal{T}_{h, i}}\|v\|_{m, T}^{2}\right)^{1 / 2}
$$


Let $E_{m}$ and $E_{n}$ be two adjacent elements in $\mathcal{T}_{h, i}$, with $n<m$, and let $\gamma=$ $\partial E_{n} \cap \partial E_{m} \in \mathcal{E}_{h, i}$ We denote the average and jump on $\gamma$ for an elementwise smooth function $\phi$ by, respectively,

$$
\begin{aligned}
\{\{\phi\} & :=\frac{1}{2}\left(\left.\left(\left.\phi\right|_{E_{n}}\right)\right|_{\gamma}+\left.\left(\left.\phi\right|_{E_{m}}\right)\right|_{\gamma}\right), \\
\llbracket \phi \rrbracket: & =\left.\left(\left.\phi\right|_{E_{n}}\right)\right|_{\gamma}-\left.\left(\phi_{E_{m}}\right)\right|_{\gamma} .
\end{aligned}
$$

On a boundary face $\left(\gamma \cap \Gamma_{D} \neq \emptyset\right.$ or $\left.\gamma \cap \Gamma_{N} \neq \emptyset\right)$ we set,

$$
\{\phi\}:=\left.\phi\right|_{\gamma}, \quad \llbracket \phi \rrbracket:=\left.\phi\right|_{\gamma} .
$$

Let $\mathcal{T}_{H, i, j}$ be a partition of $\Gamma_{i, j}$ with maximal element diameter $H$. We use $\Gamma_{H}=\cup_{1<j \leq N} \Gamma_{i, j}$ to denote the union of all the mortar grids and $\Gamma_{H, i}=\Gamma_{H} \cap \partial \Omega_{i}$ to denote the intersection of the mortar grids with the boundary of $\Omega_{i}$. We do not need to assume $\mathcal{T}_{H, i, j}$ is conforming, but we may make this assumption if we want to use continuous mortar approximations. Furthermore, we do not assume $\mathcal{T}_{H, i, j}$ aligns with either subdomain partitions $\mathcal{T}_{h, i}$ or $\mathcal{T}_{h, j}$. In the multiscale setting, the mortar grid is chosen to be much coarser than the trace of the subdomain grids, i.e., $H>>h$.

In this paper we take

$$
M_{H}^{r}=\left\{\mu \in L^{2}(\Gamma):\left.\mu\right|_{\mathcal{T}_{H, i, j}} \in \mathbb{P}_{r}(\tau), \forall \tau \in \mathcal{T}_{H, i, j}\right\}, \quad r \geq 0,
$$

or the continuous counterpart for the discrete mortar approximations. If $M_{H}^{r}$ consists of discontinuous polynomials, then we take $\mathcal{Q}_{H}$ to be the $\mathrm{L}^{2}(\gamma)$-projection on each $\gamma \in \Gamma$. If we use the continuous version of the mortar space then we take $\mathcal{Q}_{H}$ to be the $\mathrm{L}^{2}\left(\Gamma_{i, j}\right)$-projection.

7.1. Mixed Finite Elements. For $i \in I_{M}$, the mixed variational formulation of (2.3) seeks $\left(\mathbf{u}_{i}, p_{i}\right) \in \mathbf{H}\left(\operatorname{div}, \Omega_{i}\right) \times L^{2}\left(\Omega_{i}\right)$ such that,

$$
\left\{\begin{array}{l}
\left(\mathbf{K}^{-1} \mathbf{u}_{i}, \mathbf{v}_{i}\right)_{\Omega_{i}}-\left(p_{i}, \nabla \cdot \mathbf{v}_{i}\right)_{\Omega_{i}}=-\langle g, \mathbf{v} \cdot \mathbf{n}\rangle_{\partial \Omega_{i} \cap \partial \Omega}-\langle\lambda, \mathbf{v} \cdot \mathbf{n}\rangle_{\Gamma_{i}}, \\
\left(\nabla \cdot \mathbf{u}_{i}, q_{i}\right)=\left(f, q_{i}\right)_{\Omega_{i}},
\end{array}\right.
$$

for all $\left(\mathbf{v}_{i}, q_{i}\right) \in \mathbf{H}\left(\operatorname{div}, \Omega_{i}\right) \times L^{2}\left(\Omega_{i}\right)$.

Using the notation introduced in section 4 , we define

$$
\mathbf{z}_{i}=\left(\mathbf{u}_{i}, p_{i}\right), \quad \mathbf{w}_{i}=\left(\mathbf{v}_{i}, q_{i}\right), \quad \text { and } \quad \mathbf{V}_{i}=\mathbf{H}\left(\operatorname{div}, \Omega_{i}\right) \times L^{2}\left(\Omega_{i}\right) .
$$

The corresponding bilinear forms are defined as

$$
\begin{aligned}
\mathcal{A}_{i}\left(\mathbf{z}_{i}, \mathbf{w}_{i}\right) & =\left(\mathbf{K}^{-1} \mathbf{u}_{i}, \mathbf{v}_{i}\right)_{\Omega_{i}}-\left(p_{i}, \nabla \cdot \mathbf{v}_{i}\right)_{\Omega_{i}}+\left(\nabla \cdot \mathbf{u}_{i}, q_{i}\right) \\
\mathcal{B}_{i}\left(\lambda, \mathbf{w}_{i}\right) & =\langle\lambda, \mathbf{v} \cdot \mathbf{n}\rangle_{\Gamma_{i}}, \\
l_{i}\left(\mathbf{w}_{i}\right) & =\left(f, q_{i}\right)_{\Omega_{i}}+\langle g, \mathbf{v} \cdot \mathbf{n}\rangle_{\partial \Omega_{i} \cap \partial \Omega}, \\
\mathcal{C}_{i}\left(\mathbf{z}_{i}, \mu\right) & =-\left\langle\mathbf{u}_{i} \cdot \mathbf{n}, \mu\right\rangle_{\Gamma_{i}}, \\
\mathcal{D}_{i}(\lambda, \mu) & =0 .
\end{aligned}
$$

To discretize, we let $\mathbf{V}_{h, i} \subset \mathbf{H}\left(\operatorname{div}, \Omega_{i}\right) \times L^{2}\left(\Omega_{i}\right)$ be any of the Raviart-Thomas $\left(\mathbf{R T}^{k}\right)$ finite element spaces $[15,49]$. The projection operator is given by $\boldsymbol{\Pi}_{h, i}:=$ $\pi_{h, i} \times \mathcal{Q}_{h, i}$ where $\pi_{h, i}$ is the well-known projection operator $[7,4]$ for the velocities and $\mathcal{Q}_{h, i}$ is the standard $\mathrm{L}^{2}(T)$-projection operator for the scalar potential for any $T \in \mathcal{T}_{h, i}$. 
Meanwhile, $i \in I_{M}$, the mixed variational formulation of (3.3) seeks $\left(\mathbf{s}_{i}, \xi_{i}\right) \in$ $\mathbf{H}\left(\operatorname{div}, \Omega_{i}\right) \times L^{2}\left(\Omega_{i}\right)$ such that,

$$
\left\{\begin{array}{l}
\left(\mathbf{K}^{-1} \mathbf{s}_{i}, \mathbf{v}_{i}\right)_{\Omega_{i}}+\left(\xi_{i}, \nabla \cdot \mathbf{v}_{i}\right)_{\Omega_{i}}=\langle\eta, \mathbf{v} \cdot \mathbf{n}\rangle_{\Gamma_{i}}, \\
-\left(\nabla \cdot \mathbf{s}_{i}, q_{i}\right)=\left(\psi, q_{i}\right)_{\Omega_{i}},
\end{array}\right.
$$

for all $\left(\mathbf{v}_{i}, q_{i}\right) \in \mathbf{H}\left(\operatorname{div}, \Omega_{i}\right) \times L^{2}\left(\Omega_{i}\right)$.

Using the notation introduced in section 4.2, we define

$$
\phi_{i}=\left(\mathbf{s}_{i}, \xi_{i}\right), \quad \mathbf{w}_{i}=\left(\mathbf{v}_{i}, q_{i}\right), \quad \text { and } \quad \mathbf{V}_{i}=\mathbf{H}\left(\operatorname{div}, \Omega_{i}\right) \times L^{2}\left(\Omega_{i}\right) .
$$

The corresponding adjoint bilinear forms are defined as

$$
\begin{aligned}
\mathcal{A}_{i}^{*}\left(\phi_{i}, \mathbf{w}_{i}\right) & =\left(\mathbf{K}^{-1} \mathbf{s}_{i}, \mathbf{v}_{i}\right)_{\Omega_{i}}+\left(\xi_{i}, \nabla \cdot \mathbf{v}_{i}\right)_{\Omega_{i}}-\left(\nabla \cdot \mathbf{s}_{i}, q_{i}\right) \\
\mathcal{C}_{i}^{*}\left(\eta, \mathbf{w}_{i}\right) & =-\langle\eta, \mathbf{v} \cdot \mathbf{n}\rangle_{\Gamma_{i}}, \\
j_{i}\left(\mathbf{w}_{i}\right) & =\left(\psi, q_{i}\right)_{\Omega_{i}} \\
\mathcal{B}_{i}^{*}\left(\phi_{i}, \mu\right) & =\left\langle\mathbf{s}_{i} \cdot \mathbf{n}, \mu\right\rangle_{\Gamma_{i}}, \\
\mathcal{D}_{i}^{*}(\eta, \mu) & =0
\end{aligned}
$$

Notice that, due to our choice of adjoint flux, all of the bilinear forms are adjoint consistent for the mixed formulation, i.e., both (4.11) and (4.12) hold. The terms in the error representation corresponding to $i \in I_{M}$ are easily seen to be

$$
\begin{aligned}
\mathcal{E}_{\mathrm{sub}, i}= & \left(f, \xi_{i}-\mathcal{Q}_{h, i} \xi_{i}\right)_{\Omega_{i}}+\left\langle g_{D},\left(\mathbf{s}_{i}-\pi_{h, i} \mathbf{s}_{i}\right) \cdot \mathbf{n}\right\rangle_{\partial \Omega_{i} \cap \partial \Omega} \\
& -\left(\mathbf{K}^{-1} \mathbf{u}_{h, i}, \mathbf{s}_{i}-\pi_{h, i} \mathbf{s}_{i}\right)_{\Omega_{i}}+\left(p_{h, i}, \nabla \cdot\left(\mathbf{s}_{i}-\pi_{h, i} \mathbf{s}_{i}\right)\right)_{\Omega_{i}} \\
& -\left(\nabla \cdot \mathbf{u}_{h, i}, \xi_{i}-\mathcal{Q}_{h, i} \xi_{i}\right)-\left\langle\lambda_{H},\left(\mathbf{s}_{i}-\pi_{h, i} \mathbf{s}_{i}\right) \cdot \mathbf{n}\right\rangle_{\Gamma_{i}}, \\
\mathcal{E}_{\text {mort }, i}= & \left\langle\mathbf{u}_{h, i} \cdot \mathbf{n}, \eta-\mathcal{Q}_{H} \eta\right\rangle_{\Gamma_{i}}, \\
\mathcal{E}_{\text {cons }, i}= & 0
\end{aligned}
$$

7.2. Discontinuous and Continuous Galerkin. For $i \in I_{P}$, we set

$$
\mathbf{z}_{i}=p_{i}, \quad \mathbf{w}_{i}=q_{i}, \quad \text { and } \quad \mathbf{V}_{i}=H^{1}\left(\mathcal{T}_{h, i}\right)
$$

The interior penalty discontinuous Galerkin formulation of $(2.2)$ seeks $p_{i} \in H^{1}\left(\mathcal{T}_{h, i}\right)$ such that,

$$
\mathcal{A}_{i}\left(\mathbf{z}_{i}, \mathbf{w}_{i}\right)=l_{i}\left(\mathbf{w}_{i}\right)-\mathcal{B}_{i}\left(\lambda, \mathbf{w}_{i}\right), \quad \forall \mathbf{w}_{i} \in \mathbf{V}_{i}
$$


where

$$
\begin{aligned}
\mathcal{A}_{i}\left(\mathbf{z}_{i}, \mathbf{w}_{i}\right)= & \sum_{T \in \mathcal{T}_{h, i}}\left(\mathbf{K} \nabla p_{i}, \nabla q_{i}\right)_{T} \\
& +\sum_{\gamma \in \mathcal{E}_{h, i}}\left(-\left\langle\left\{\mathbf{K} \nabla p_{i} \cdot \mathbf{n}\right\}, \llbracket q_{i} \rrbracket\right\rangle_{\gamma}-s_{\mathrm{f}}\left\langle\left\{\mathbf{K} \nabla q_{i} \cdot \mathbf{n}\right\}, \llbracket p_{i} \rrbracket\right\rangle_{\gamma}+\frac{\sigma_{\gamma}}{h_{\gamma}}\left\langle\llbracket p_{i} \rrbracket, \llbracket q_{i} \rrbracket\right\rangle_{\gamma}\right) \\
& +\sum_{\gamma \in \mathcal{E}_{h, i}^{\text {ext }}}\left(-\left\langle\mathbf{K} \nabla p_{i} \cdot \mathbf{n}, q_{i}\right\rangle_{\gamma}-\left\langle\mathbf{K} \nabla q_{i} \cdot \mathbf{n}, p_{i}\right\rangle_{\gamma}+\frac{\sigma_{\gamma}}{h_{\gamma}}\left\langle p_{i}, q_{i}\right\rangle_{\gamma}\right) \\
& +\sum_{\gamma \in \mathcal{E}_{h, i}^{\Gamma}}\left(-\left\langle\mathbf{K} \nabla p_{i} \cdot \mathbf{n}, q_{i}\right\rangle_{\gamma}-\left\langle\mathbf{K} \nabla q_{i} \cdot \mathbf{n}, p_{i}\right\rangle_{\gamma}+\frac{\sigma_{\gamma}}{h_{\gamma}}\left\langle p_{i}, q_{i}\right\rangle_{\gamma}\right) \\
l_{i}\left(\mathbf{w}_{i}\right)= & \sum_{T \in \mathcal{T}_{h, i}}\left(f, q_{i}\right)_{T_{i}}+\sum_{\gamma \in \mathcal{E}_{h, i}^{\text {ext }}}\left(-\left\langle\mathbf{K} \nabla q_{i} \cdot \mathbf{n}_{i}, g_{D}\right\rangle_{\gamma}+\frac{\sigma_{\gamma}}{h_{\gamma}}\left\langle g_{D}, q_{i}\right\rangle_{\gamma}\right), \\
\mathcal{B}_{i}\left(\lambda, \mathbf{w}_{i}\right)= & \sum_{\gamma \in \mathcal{E}_{h, i}^{\Gamma}}\left(\left\langle\mathbf{K} \nabla q_{i} \cdot \mathbf{n}_{i}, \lambda\right\rangle_{\gamma}-\frac{\sigma_{\gamma}}{h_{\gamma}}\left\langle\lambda, q_{i}\right\rangle_{\gamma}\right) .
\end{aligned}
$$

The remaining bilinear forms are given by,

$$
\begin{aligned}
& \mathcal{C}_{i}\left(\mathbf{z}_{i}, \mu\right)=\sum_{\gamma \in \mathcal{E}_{h, i}^{\Gamma}}\left(\left\langle\mathbf{K} \nabla p_{i} \cdot \mathbf{n}_{i}, \mu\right\rangle_{\gamma}-\frac{\sigma_{\gamma}}{h_{\gamma}}\left\langle p_{i}, \mu\right\rangle_{\gamma}\right), \\
& \mathcal{D}_{i}(\lambda, \mu)=\sum_{\gamma \in \mathcal{E}_{h, i}^{\Gamma}} \frac{\sigma_{\gamma}}{h_{\gamma}}\langle\lambda, \mu\rangle_{\gamma} .
\end{aligned}
$$

Various interior penalty DG methods can be obtained by a proper choice of the form parameter, $s_{\mathrm{f}}$. If we choose $s_{\mathrm{f}}=1$, then $\mathcal{A}_{i}(\cdot, \cdot)$ will be symmetric and we recover the symmetric interior penalty Galerkin (SIPG) method. Similarly, setting $s_{\mathrm{f}}=0$ and $s_{\mathrm{f}}=-1$ give the incomplete (IIPG) and the nonsymmetric (NIPG) interior penalty Galerkin methods respectively. The penalty parameter, $\sigma_{\gamma}$ is taken to be a constant value on each element face and we assume that $0<\sigma^{0} \leq \sigma_{\gamma} \leq \sigma^{1}$.

We fix the form parameter to be 1 along the boundary edges which preserves the symmetry of the formulation if SIPG is used. This choice forces us to use $h_{\gamma}$ rather than $H_{\gamma}$ in the penalty terms along $\mathcal{E}_{h, i}^{\Gamma}$ which results in slightly suboptimal a priori convergence rates in the multiscale case [35], i.e., if $H=\mathcal{O}\left(h^{\alpha}\right)$ with $\alpha<1$.

We discretize by choosing $\mathbf{V}_{h, i}=\mathbb{P}_{k}\left(\mathcal{T}_{h, i}\right)$, the space of polynomials of degree $k$ on $T \in \mathcal{T}_{h, i}$, with $k \geq 1$. If $\sigma$ if sufficiently large, then the standard DG arguments can be used to show discrete stability and to derive a priori error bounds [35]. The projection operator is defined to be $\boldsymbol{\Pi}_{h, i}=\mathcal{Q}_{h, i}$ where $\mathcal{Q}_{h, i}$ is the local L ${ }^{2}(T)$-projection.

Meanwhile, using the notation introduced in section 4, we define

$$
\mathbf{z}_{i}=p_{i}, \quad \mathbf{w}_{i}=q_{i}, \quad \text { and } \quad \mathbf{V}_{i}=H^{1}\left(\mathcal{T}_{h, i}\right)
$$

The interior penalty discontinuous Galerkin formulation of $(3.2)$ seeks $\xi_{i} \in H^{1}\left(\mathcal{T}_{h, i}\right)$ such that

$$
\mathcal{A}_{i}^{*}\left(\phi_{i}, \mathbf{w}_{i}\right)=j_{i}\left(\mathbf{w}_{i}\right)-\mathcal{B}_{i}^{*}\left(\eta, \mathbf{w}_{i}\right), \quad \forall \mathbf{w}_{i} \in \mathbf{V}_{i},
$$


where

$$
\begin{aligned}
\mathcal{A}_{i}^{*}\left(\boldsymbol{\phi}_{i}, \mathbf{w}_{i}\right)= & \sum_{T \in \mathcal{T}_{h, i}}\left(\mathbf{K} \nabla \xi_{i}, \nabla q_{i}\right)_{T} \\
& +\sum_{\gamma \in \mathcal{E}_{h, i}}\left(-\left\langle\left\{\mathbf{K} \nabla \xi_{i} \cdot \mathbf{n} \rrbracket, \llbracket q_{i} \rrbracket\right\rangle_{\gamma}-s_{\mathrm{f}}^{*}\left\langle\left\{\mathbf{K} \nabla q_{i} \cdot \mathbf{n} \rrbracket, \llbracket \xi_{i} \rrbracket\right\rangle_{\gamma}+\frac{\sigma_{\gamma}}{h_{\gamma}}\left\langle\llbracket \xi_{i} \rrbracket, \llbracket q_{i} \rrbracket\right\rangle_{\gamma}\right)\right.\right. \\
& +\sum_{\gamma \in \mathcal{E}_{h, i}^{\text {ext }}}\left(-\left\langle\mathbf{K} \nabla \xi_{i} \cdot \mathbf{n}, q_{i}\right\rangle_{\gamma}-\left\langle\mathbf{K} \nabla q_{i} \cdot \mathbf{n}, \xi_{i}\right\rangle_{\gamma}+\frac{\sigma_{\gamma}}{h_{\gamma}}\left\langle\xi_{i}, q_{i}\right\rangle_{\gamma}\right) \\
& +\sum_{\gamma \in \mathcal{E}_{h, i}^{\Gamma}}\left(-\left\langle\mathbf{K} \nabla \xi_{i} \cdot \mathbf{n}, q_{i}\right\rangle_{\gamma}-\left\langle\mathbf{K} \nabla q_{i} \cdot \mathbf{n}, \xi_{i}\right\rangle_{\gamma}+\frac{\sigma_{\gamma}}{h_{\gamma}}\left\langle\xi_{i}, q_{i}\right\rangle_{\gamma}\right) \\
j_{i}\left(\mathbf{w}_{i}\right)= & \sum_{T \in \mathcal{T}_{h, i}}\left(\psi, q_{i}\right)_{T_{i}}, \\
\mathcal{C}_{i}^{*}\left(\eta, \mathbf{w}_{i}\right)= & \sum_{\gamma \in \mathcal{E}_{h, i}^{\Gamma}}\left(\left\langle\mathbf{K} \nabla q_{i} \cdot \mathbf{n}_{i}, \eta\right\rangle_{\gamma}-\frac{\sigma_{\gamma}}{h_{\gamma}}\left\langle\eta, q_{i}\right\rangle_{\gamma}\right) .
\end{aligned}
$$

The remaining adjoint bilinear forms are given by,

$$
\begin{aligned}
\mathcal{B}_{i}^{*}\left(\phi_{i}, \mu\right) & =\sum_{\gamma \in \mathcal{E}_{h, i}^{\Gamma}}\left(\left\langle\mathbf{K} \nabla \xi_{i} \cdot \mathbf{n}_{i}, \mu\right\rangle_{\gamma}-\frac{\sigma_{\gamma}}{h_{\gamma}}\left\langle\xi_{i}, \mu\right\rangle_{\gamma}\right), \\
\mathcal{D}_{i}^{*}(\eta, \mu) & =\sum_{\gamma \in \mathcal{E}_{h, i}^{\Gamma}} \frac{\sigma_{\gamma}}{h_{\gamma}}\langle\eta, \mu\rangle_{\gamma} .
\end{aligned}
$$

Notice that (4.12) holds due to the definition of the bilinear forms and our choice of the form parameter along the boundaries. However, (4.11) does not hold if NIPG or IIPG methods are used. Fortunately, it was shown in [57] that,

$$
\mathcal{E}_{\text {cons }, i}=\mathcal{A}_{i}\left(\mathbf{e}_{z, i}, \phi_{i}\right)-\mathcal{A}_{i}^{*}\left(\phi_{i}, \mathbf{e}_{z, i}\right)=\sum_{\gamma \in \mathcal{E}_{h, i}}\left(-1+s_{\mathrm{f}}\right)\left\langle\left\{\left\{\mathbf{K} \nabla \xi_{i} \cdot \mathbf{n}\right\}, \llbracket p_{h, i} \rrbracket\right\rangle_{\gamma},\right.
$$

which is fully computable and does not depend on the choice of DG method used to solve the adjoint. Also note that this term is zero if SIPG is used, i.e., if the DG formulation is adjoint consistent. Using the definition of the linear and bilinear forms 
we can derive the subdomain error indicator,

$$
\begin{aligned}
\mathcal{E}_{\mathrm{sub}, i}= & \sum_{T \in \mathcal{T}_{h, i}}\left(f, \xi_{i}-\mathcal{Q}_{h, i} \xi_{i}\right)_{T_{i}}-\sum_{T \in \mathcal{T}_{h, i}}\left(\mathbf{K} \nabla p_{h, i}, \nabla\left(\xi_{i}-\mathcal{Q}_{h, i} \xi_{i}\right)\right)_{T} \\
& +\sum_{\gamma \in \mathcal{E}_{h, i}^{\text {ext }}}\left(-\left\langle\mathbf{K} \nabla\left(\xi_{i}-\mathcal{Q}_{h, i} \xi_{i}\right) \cdot \mathbf{n}_{i}, g_{D}\right\rangle_{\gamma}+\frac{\sigma_{\gamma}}{h_{\gamma}}\left\langle g_{D}, \xi_{i}-\mathcal{Q}_{h, i} \xi_{i}\right\rangle_{\gamma}\right) \\
& -\sum_{\gamma \in \mathcal{E}_{h, i}}\left(-\left\langle\left\{\mathbf{K} \nabla p_{h, i} \cdot \mathbf{n}\right\}\right\}, \llbracket \xi_{i}-\mathcal{Q}_{h, i} \xi_{i} \rrbracket\right\rangle_{\gamma}-s_{\mathrm{f}}\left\langle\left\{\mathbf{K} \nabla\left(\xi_{i}-\mathcal{Q}_{h, i} \xi_{i}\right) \cdot \mathbf{n} \rrbracket, \llbracket p_{h, i} \rrbracket\right\rangle_{\gamma}\right. \\
& \left.+\frac{\sigma_{\gamma}}{h_{\gamma}}\left\langle\llbracket p_{h, i} \rrbracket, \llbracket \xi_{i}-\mathcal{Q}_{h, i} \xi_{i} \rrbracket\right\rangle_{\gamma}\right) \\
& -\sum_{\gamma \in \mathcal{E}_{h, i}^{\text {ext }}}\left(-\left\langle\mathbf{K} \nabla p_{h, i} \cdot \mathbf{n}, \xi_{i}-\mathcal{Q}_{h, i} \xi_{i}\right\rangle_{\gamma}-\left\langle\mathbf{K} \nabla\left(\xi_{i}-\mathcal{Q}_{h, i} \xi_{i}\right) \cdot \mathbf{n}, p_{h, i}\right\rangle_{\gamma}\right. \\
& \left.+\frac{\sigma_{\gamma}}{h_{\gamma}}\left\langle p_{h, i}, \xi_{i}-\mathcal{Q}_{h, i} \xi_{i}\right\rangle_{\gamma}\right) \\
& -\sum_{\gamma \in \mathcal{E}_{h, i}^{\Gamma}}\left(-\left\langle\mathbf{K} \nabla p_{h, i} \cdot \mathbf{n}, \xi_{i}-\mathcal{Q}_{h, i} \xi_{i}\right\rangle_{\gamma}-\left\langle\mathbf{K} \nabla\left(\xi_{i}-\mathcal{Q}_{h, i} \xi_{i}\right) \cdot \mathbf{n}, p_{h, i}\right\rangle_{\gamma}\right. \\
& \left.+\frac{\sigma_{\gamma}}{h_{\gamma}}\left\langle p_{h, i}, \xi_{i}-\mathcal{Q}_{h, i} \xi_{i}\right\rangle_{\gamma}\right) \\
& -\sum_{\gamma \in \mathcal{E}_{h, i}^{\Gamma}}\left(\left\langle\mathbf{K} \nabla\left(\xi_{i}-\mathcal{Q}_{h, i} \xi_{i}\right) \cdot \mathbf{n}_{i}, \lambda_{H}\right\rangle_{\gamma}-\frac{\sigma_{\gamma}}{h_{\gamma}}\left\langle\lambda_{H}, \xi_{i}-\mathcal{Q}_{h, i} \xi_{i}\right\rangle_{\gamma}\right)
\end{aligned}
$$

and the mortar error indicator,

$$
\begin{aligned}
\mathcal{E}_{\text {mort }, i}= & -\sum_{\gamma \in \mathcal{E}_{h, i}^{\Gamma}}\left(\left\langle\mathbf{K} \nabla p_{h, i} \cdot \mathbf{n}_{i}, \eta-\mathcal{Q}_{H} \eta\right\rangle_{\gamma}-\frac{\sigma_{\gamma}}{h_{\gamma}}\left\langle p_{h, i}, \eta-\mathcal{Q}_{H} \eta\right\rangle_{\gamma}\right), \\
& -\sum_{\gamma \in \mathcal{E}_{h, i}^{\Gamma}} \frac{\sigma_{\gamma}}{h_{\gamma}}\left\langle\lambda_{H}, \eta-\mathcal{Q}_{H} \eta\right\rangle_{\gamma}
\end{aligned}
$$

REMARK 7.1. We may also consider a continuous Galerkin (CG) formulation for $i \in I_{p}$ with weakly imposed Dirichlet conditions by taking

$$
\mathbf{V}_{h, i}:=\left\{v \in C\left(\Omega_{i}\right) \cap H^{1}\left(\Omega_{i}\right): \forall T \in \mathcal{T}_{h, i},\left.v\right|_{T} \in \mathbb{P}^{k}(T)\right\} .
$$

The subdomain bilinear and linear forms simplify to,

$$
\begin{aligned}
\mathcal{A}_{i}\left(\mathbf{z}_{i}, \mathbf{w}_{i}\right)= & \left(\mathbf{K} \nabla p_{i}, \nabla q_{i}\right)_{\Omega_{i}}+\sum_{\gamma \in \mathcal{E}_{h, i}^{\text {ext }}}\left(-\left\langle\mathbf{K} \nabla p_{i} \cdot \mathbf{n}, q_{i}\right\rangle_{\gamma}-\left\langle\mathbf{K} \nabla q_{i} \cdot \mathbf{n}, p_{i}\right\rangle_{\gamma}+\frac{\sigma_{\gamma}}{h_{\gamma}}\left\langle p_{i}, q_{i}\right\rangle_{\gamma}\right) \\
& +\sum_{\gamma \in \mathcal{E}_{h, i}^{\Gamma}}\left(-\left\langle\mathbf{K} \nabla p_{i} \cdot \mathbf{n}, q_{i}\right\rangle_{\gamma}-\left\langle\mathbf{K} \nabla q_{i} \cdot \mathbf{n}, p_{i}\right\rangle_{\gamma}+\frac{\sigma_{\gamma}}{h_{\gamma}}\left\langle p_{i}, q_{i}\right\rangle_{\gamma}\right) \\
l_{i}\left(\mathbf{w}_{i}\right)= & \left(f, q_{i}\right)_{\Omega_{i}}+\sum_{\gamma \in \mathcal{E}_{h, i}^{\text {ext }}}\left(-\left\langle\mathbf{K} \nabla q_{i} \cdot \mathbf{n}_{i}, g_{D}\right\rangle_{\gamma}+\frac{\sigma_{\gamma}}{h_{\gamma}}\left\langle g_{D}, q_{i}\right\rangle_{\mathcal{E}_{h, i}^{\text {ext }}}\right) .
\end{aligned}
$$

The other linear and bilinear forms remain the same, but the projection operator becomes either the global $L^{2}\left(\Omega_{i}\right)$-projection or the interpolant. Most of the terms in the error representation are the same, except for the terms on the interior edges $\left(\gamma \in \mathcal{E}_{h, i}\right)$, which are zero. 
8. Numerical Results. Consider the model elliptic problem (2.1) on $\Omega=$ $(0,1) \times(0,1)$ with

$$
\mathbf{K}(x, y)=(1+0.8 \sin (4 \pi x) \cos (3 \pi y)) \mathbb{I} .
$$

The boundary data and source function are chosen so that,

$$
u(x, y)=10 x(1-x) y(1-y) \exp (\sin (3 \pi x) \sin (3 \pi y)) .
$$

We consider the following quantities of interest:

1. The value of the solution at $(1 / 3,1 / 3)$ for which

$$
\psi=\delta_{(1 / 3,1 / 3)} \approx \frac{200}{\pi} \exp \left(-200(x-1 / 3)^{2}-200(y-1 / 3)^{2}\right)
$$

2. The average value of the solution over $\Omega_{16}=(3 / 4,1) \times(3 / 4,1)$ for which

$$
\psi= \begin{cases}1 /\left|\Omega_{16}\right|, & x \in \Omega_{16}, \\ 0, & \text { otherwise }\end{cases}
$$

3. The average value of the mortar solution along $\Gamma_{6,7}$ where $\Omega_{6}=(1 / 4,1 / 2) \times$ $(1 / 4,1 / 2)$ and $\Omega_{7}=(1 / 2,3 / 4) \times(1 / 4,1 / 2)$ for which

$$
\eta_{M}= \begin{cases}1 /\left|\Gamma_{6,7}\right|, & x \in \Gamma_{6,7}, \\ 0, & \text { otherwise }\end{cases}
$$

In all of the numerical examples, we use a decomposition of $\Omega$ into 16 equal-sized subdomains arranged in a $4 \times 4$ pattern. In Figure 8.1, we plot the sets of grids to be used in sections 8.1 and 8.2. We allow triangular or quadrilateral elements in
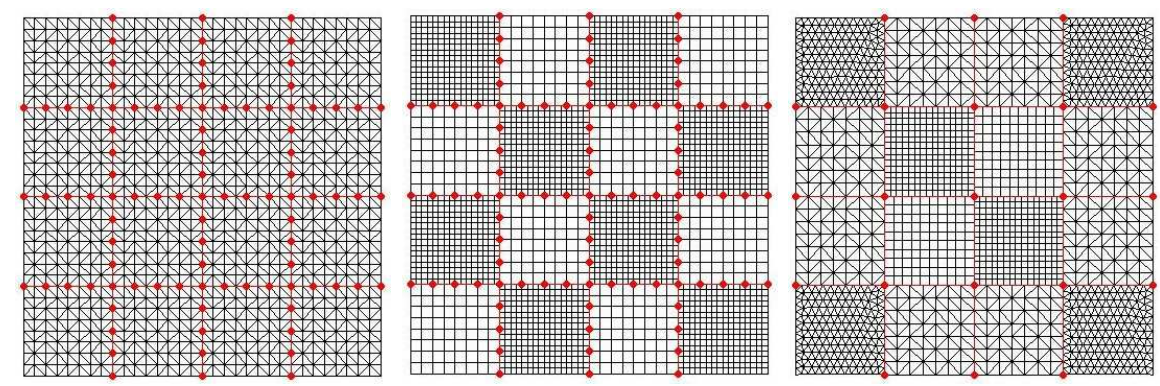

FIG. 8.1. Subdomain and mortar grids. From left to right: grid (a), grid (b) and grid (c).

each subdomain. Note that only grid (a) aligns along the interface. Grids containing both triangular and rectangular elements may also be considered using mixed and DG approximations [41], but this is an unnecessary complication for this paper. In all three cases, the mortar mesh does not align with any of the subdomain grids. Grid (c) is designed for the multiscale mortar setting where we allow only one mortar element on each interface.

8.1. Mononumerics Coupling. In this section, we use the same discretization method for all of the subdomains and compute the a posteriori error estimate using a higher order approximation of the same type. We consider the following subdomain approximations: 
- $\mathrm{RT}^{0}$ mixed finite elements for the forward problem and $\mathrm{RT}^{1}$ for the adjoint problem.

- Piecewise linear $\left(\mathbb{P}_{1}\left(\mathcal{T}_{h, i}\right)\right)$ NIPG for the forward problem and piecewise quadratic $\left(\mathbb{P}_{2}\left(\mathcal{T}_{h, i}\right)\right)$ NIPG for the adjoint problem.

- Continuous piecewise linear Galerkin for the forward problem and continuous piecewise quadratic for the adjoint problem.

We consider the following cases:

1. Quantity of interest 1, on grid (a), with discontinuous linear mortars.

2. Quantity of interest 2, on grid (b), with discontinuous linear mortars.

3. Quantity of interest 3, on grid (c), with discontinuous cubic mortars.

Other cases were considered, e.g., computing all three quantities of interest on all three grids, but these cases yielded similar results and were omitted in the interest of space. In each case, we solve the subdomain problems using a sparse direct solver. We construct the forward and adjoint interface operators (4.17) and (4.19) and use GMRES to solve the interface problem to a tolerance of $1 \mathrm{E}-10$.

In Figure 8.2, we plot the subdomain adjoint solutions (top row) and the adjoint mortar solutions (bottom row) for each quantity of interest using NIPG to solve each of the three adjoint problems (second of the three subdomain approximation choices).
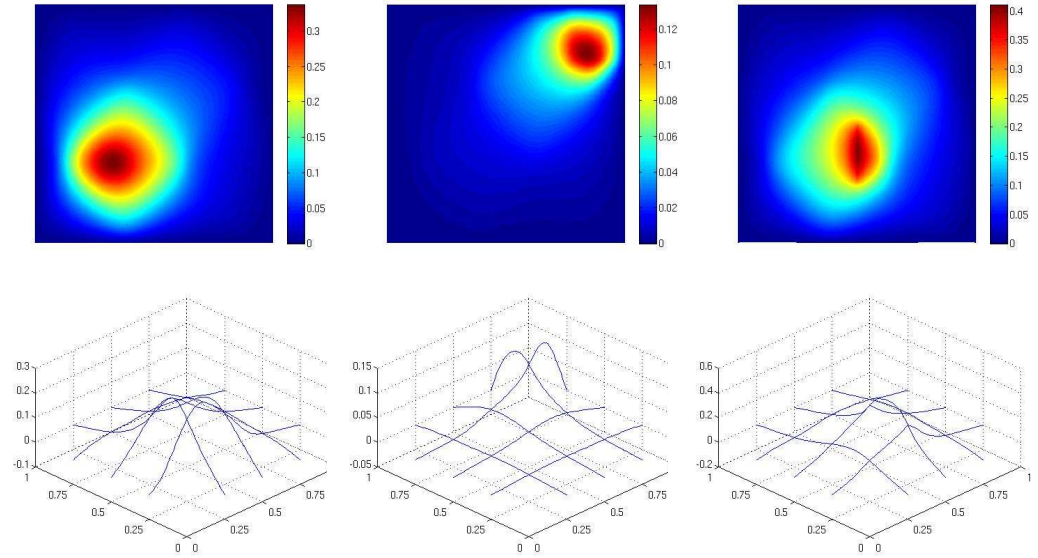

FIG. 8.2. The subdomain adjoint solutions (top row) and the adjoint mortar solutions (bottom row) for each quantity of interest using NIPG to solve the adjoint problems.

In Table 8.1, we present the subdomain, mortar and consistency terms of the a posteriori error estimate along with the true error in the quantity of interests and effectivity ratios. In all nine simulations, the effectivity ratio is close to one, indicating that the a posteriori error estimate is quite accurate even with an approximate (numerical) adjoint. The consistency error is nonzero only for NIPG and is approximately the same order of magnitude as the other terms in the error estimate. We also note that the mortar error using mixed finite elements for case 1 is nearly zero. This is due to the fact that the mortar space contained the same number of degrees of freedom as the trace of the subdomain velocity space. Thus, conservation can be enforced up to the interface solver tolerance.

8.2. Multinumerics Coupling. In this section, we use grid (c) from Figure 8.1 and use either DG (SIPG), CG, or a mixed method in each subdomain as shown in 


\begin{tabular}{c|c||c|c|c|c|c}
\hline QofI & Method & $\mathcal{E}_{\text {sub }}$ & $\mathcal{E}_{\text {mort }}$ & $\mathcal{E}_{\text {cons }}$ & True Error & Effectivity \\
\hline \hline 1 & NIPG & $1.8631 \mathrm{E}-3$ & $-4.3305 \mathrm{E}-4$ & $-5.8977 \mathrm{E}-4$ & $8.3199 \mathrm{E}-4$ & 1.010 \\
\hline 2 & NIPG & $4.5324 \mathrm{E}-4$ & $1.2107 \mathrm{E}-4$ & $-3.6120 \mathrm{E}-4$ & $2.0989 \mathrm{E}-4$ & 1.015 \\
\hline 3 & NIPG & $-1.5282 \mathrm{E}-3$ & $5.7833 \mathrm{E}-4$ & $-1.4538 \mathrm{E}-4$ & $-1.0528 \mathrm{E}-3$ & 1.040 \\
\hline 1 & CG & $2.0955 \mathrm{E}-3$ & $-4.3337 \mathrm{E}-4$ & 0 & $1.6596 \mathrm{E}-3$ & 1.002 \\
\hline 2 & CG & $4.5450 \mathrm{E}-4$ & $1.2209 \mathrm{E}-4$ & 0 & $5.7529 \mathrm{E}-4$ & 1.002 \\
\hline 3 & CG & $-1.5882 \mathrm{E}-3$ & $5.8451 \mathrm{E}-4$ & 0 & $-9.5946 \mathrm{E}-4$ & 1.046 \\
\hline 1 & Mixed & $-6.8324 \mathrm{E}-4$ & $8.3000 \mathrm{E}-14$ & 0 & $-6.8044 \mathrm{E}-4$ & 1.004 \\
\hline 2 & Mixed & $7.9985 \mathrm{E}-4$ & $3.4224 \mathrm{E}-5$ & 0 & $8.3113 \mathrm{E}-4$ & 1.003 \\
\hline 3 & Mixed & $-2.5761 \mathrm{E}-3$ & $3.9105 \mathrm{E}-4$ & 0 & $-2.1619 \mathrm{E}-3$ & 1.011 \\
\hline
\end{tabular}

Error estimates and effectivity ratios using three different numerical methods to solve the subdomain problems for the three cases under consideration.

Figure 8.3.

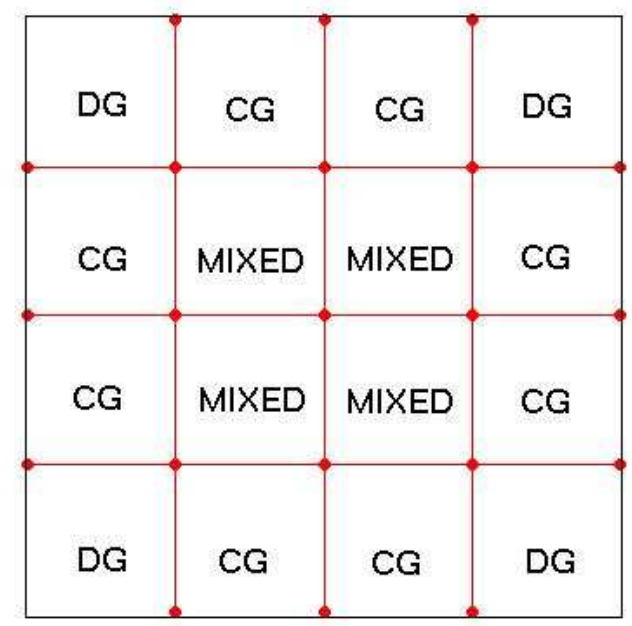

FIG. 8.3. Subdomain discretization methods used in section 8.2.

In Table 8.2, we present the components of the error estimate and the effectivity ratios for each of the three quantities of interest. The effectivity ratios are not quite

\begin{tabular}{c||c|c|c|c|c}
\hline QofI & $\mathcal{E}_{\text {sub }}$ & $\mathcal{E}_{\text {mort }}$ & $\mathcal{E}_{\text {cons }}$ & True Error & Effectivity \\
\hline \hline 1 & $-1.4960 \mathrm{E}-3$ & $-4.1564 \mathrm{E}-4$ & 0 & $-1.9166 \mathrm{E}-3$ & 0.997 \\
\hline 2 & $8.3416 \mathrm{E}-4$ & $1.4993 \mathrm{E}-6$ & 0 & $8.5018 \mathrm{E}-4$ & 0.983 \\
\hline 3 & $7.4364 \mathrm{E}-4$ & $4.1535 \mathrm{E}-4$ & 0 & $1.2002 \mathrm{E}-3$ & 0.966 \\
\hline \multicolumn{6}{c}{ TABLE 8.2}
\end{tabular}

Error estimates and effectivity ratios using three different quantities of interest using multinumerics.

as close to one as they were in the previous section, but they are still acceptable. We 
note that the consistency error is zero since we are using SIPG rather than NIPG.

8.3. Adaptive Mesh Refinement. The goal in this section is to show how the a posteriori error estimate may be used to separately adapt the mortar and subdomain grids following Alg. 1. We start with grid (a) from Figure 8.1, but we coarsen the mortars so that there is initially only one mortar element on each interface. We use piecewise linear SIPG for the subdomain discretizations and piecewise linear polynomials for the mortars. Thus, each mortar starts with only 2 degrees of freedom. The corresponding adjoint problem uses piecewise quadratic SIPG and piecewise quadratic mortars. Our quantity of interest is the average value of the solution over $\Omega_{16}$.

We refine a subdomain if its contribution is larger than $1 / 16$ of the global tolerance, which we take to be 1E-4. Similarly, we refine a mortar if its contribution is larger than $1 / 24$ of the global tolerance. Elements within a subdomain or a mortar are marked for refinement based on a similar criteria and a localization of the a posteriori error estimate.

The subdomain problems are solved using a direct factorization of the subdomain linear system using either an LU decomposition or a Cholesky factorization. For each of these iterations, we construct the coarse scale interface equation which only requires 6-10 solves per subdomain depending on the number of sides that intersect the mortar grid. The interface equation then requires 35-40 iterations of GMRES to reduce the relative residual below 1E-10. (A standard nonoverlapping domain decomposition algorithm would therefore require $35-40$ solves per subdomain. A good preconditioner, e.g., Neumann-Neumann or balancing, would reduce this number, but probably not below 6-10 since these algorithms require (at least) two subdomain solves per iteration.) Following Alg. 1, once the subdomain error is below the chosen tolerance, we build and factor the subdomain matrices and reuse these factorizations as we refine the mortar mesh.

In Figure 8.4, we plot the magnitude of each of the components of the error estimate as we adaptively refine the subdomain and mortar grids. In Table 8.3, we

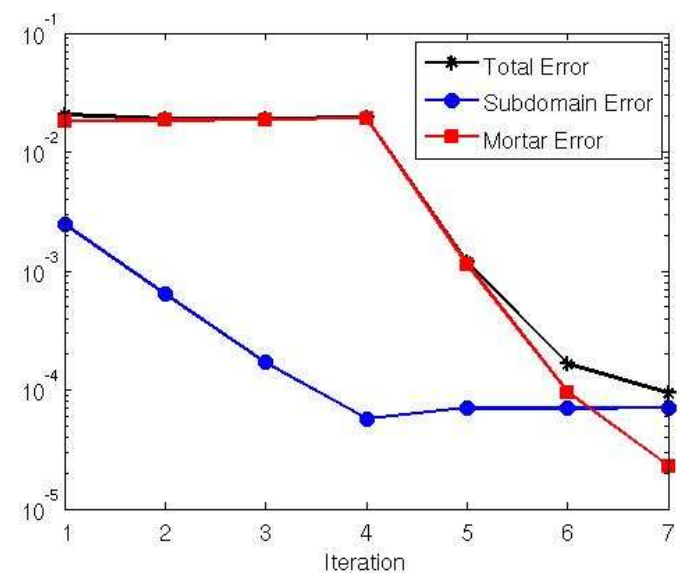

FIG. 8.4. Behavior of the components of the error estimate as the mesh iteratively refined. The first three iterations perform subdomain adaptivity while the last three perform mortar adaptivity.

give the components of the error as well as the degrees of freedom and effectivity ratios for each iteration. In Figure 8.5, we plot the final adapted subdomain and mortar grids. Further computational gains can be made by choosing a hierarchical mortar 


\begin{tabular}{c|c||c|c|c|c}
\hline Subdomain DOF & Mortar DOF & $\mathcal{E}_{\text {sub }}$ & $\mathcal{E}_{\text {mort }}$ & True Error & Effectivity \\
\hline \hline 3456 & 48 & $2.4516 \mathrm{E}-3$ & $1.8208 \mathrm{E}-2$ & $2.0547 \mathrm{E}-2$ & 1.005 \\
\hline 13824 & 48 & $6.3600 \mathrm{E}-4$ & $1.8360 \mathrm{E}-2$ & $1.8958 \mathrm{E}-2$ & 1.002 \\
\hline 42324 & 48 & $1.6961 \mathrm{E}-4$ & $1.8784 \mathrm{E}-2$ & $1.8963 \mathrm{E}-2$ & 0.999 \\
\hline 80598 & 48 & $5.7288 \mathrm{E}-5$ & $1.9245 \mathrm{E}-2$ & $1.9347 \mathrm{E}-2$ & 0.998 \\
\hline 80598 & 96 & $7.0457 \mathrm{E}-5$ & $1.1422 \mathrm{E}-3$ & $1.1901 \mathrm{E}-3$ & 1.019 \\
\hline 80598 & 158 & $7.0402 \mathrm{E}-5$ & $9.4941 \mathrm{E}-5$ & $1.6574 \mathrm{E}-4$ & 0.998 \\
\hline 80598 & 194 & $7.0538 \mathrm{E}-5$ & $2.2703 \mathrm{E}-5$ & $9.4100 \mathrm{E}-5$ & 0.991 \\
\hline \multicolumn{5}{|c}{ TABLE 8.3}
\end{tabular}

Error estimates, degrees of freedom and effectivity ratios for each level of adaptive mesh refinement. The first three iterations perform subdomain refinement and the last three perform mortar refinement.
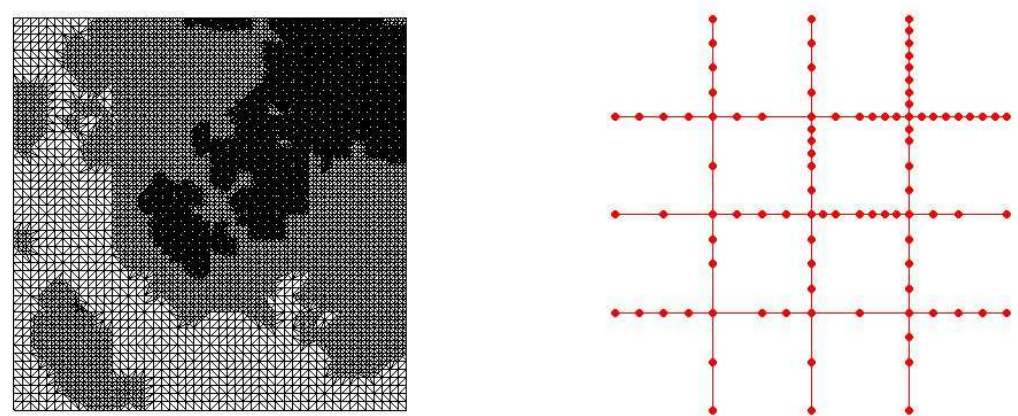

FiG. 8.5. Final adaptive subdomain (left) and mortar (right) grids.

space which would significantly reduce the cumulative number of subdomain solves, but this is beyond the scope of this paper.

8.4. Application to Flow in Porous Media. Our last example considers a practical application to flow in porous media. We consider layer 75 of the SPE-10 permeability field [17] (see Figure 8.6) and focus on mortar adaptivity. The computational domain is $\Omega=[0,1200] \times[0,2200]$. The permeability field is highly heterogeneous and is defined on a $60 \times 220$ computational grid. We divide the domain into 55 subdomains $(5 \times 11)$ as shown in Figure 8.6. Pressure-specified injection wells are placed four corner subdomains and a pressure-specified production well is placed in one of the middle subdomains. Most of the subdomains use uniform rectangular elements determined by the fine scale permeability field. In these subdomains we use mixed finite elements, or equivalently, cell-centered finite volumes. The subdomains containing wells are discretized using unstructured meshes with triangular elements which allows us to include the geometry of the well in our model. This provides an alternative to a Peaceman approximation of a pressure-specified well [40]. A cell-centered finite volume approximation is not appropriate for these computational grids, so we use a discontinuous Galerkin (SIPG) approximation. We set the pressure to be 1 at the injection wells and 0 at the production well. These values are simply for numerical demonstration and more physically relevant values can also be chosen. No-flux conditions are imposed on the outer boundaries of the domain.

Our quantity of interest is the average flux into the production well, so we set $\psi=$ 

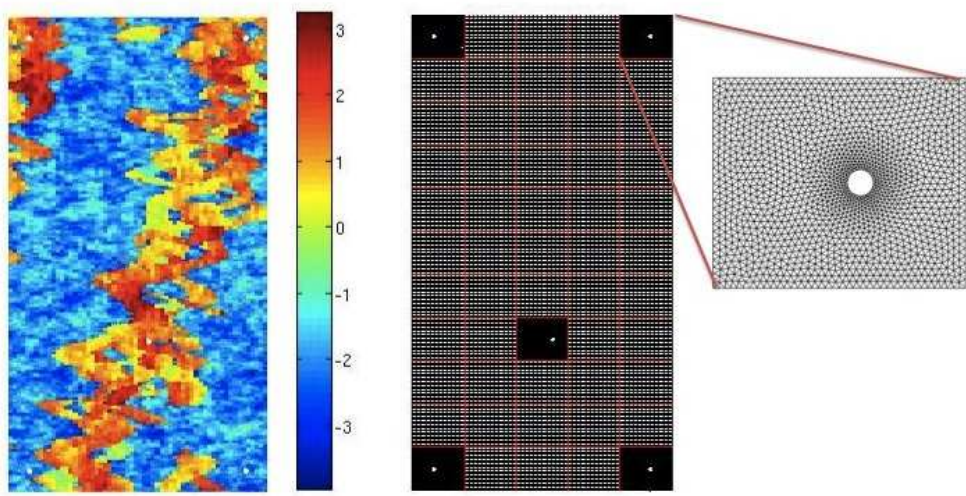

FIG. 8.6. Layer 75 of the SPE-10 permeability field (log scale) and the decomposition of the computational domain into subdomains with unstructured grids comforming to the geometry of the wells.

0 and $\xi=1 / 2 \pi r$ on the boundary of the production well where $r$ is the radius of the well. Homogeneous boundary conditions are chosen on the injection wells (Dirichlet) and on the exterior boundaries (Neumann). We assume that the subdomain grids are fixed and refine the mortar spaces until the mortar error is below a chosen tolerance. Initially, the each interface has only one mortar element with piecewise discontinuous linear mortar approximation. As in previous examples, the mortars are refined based on the localization of the a posteriori error estimate. In Figure 8.7, we plot the final adaptive mortar grid as well as the pressure field and the adjoint pressure field using this final mortar grid. We see that the mortar grid concentrates refinement between
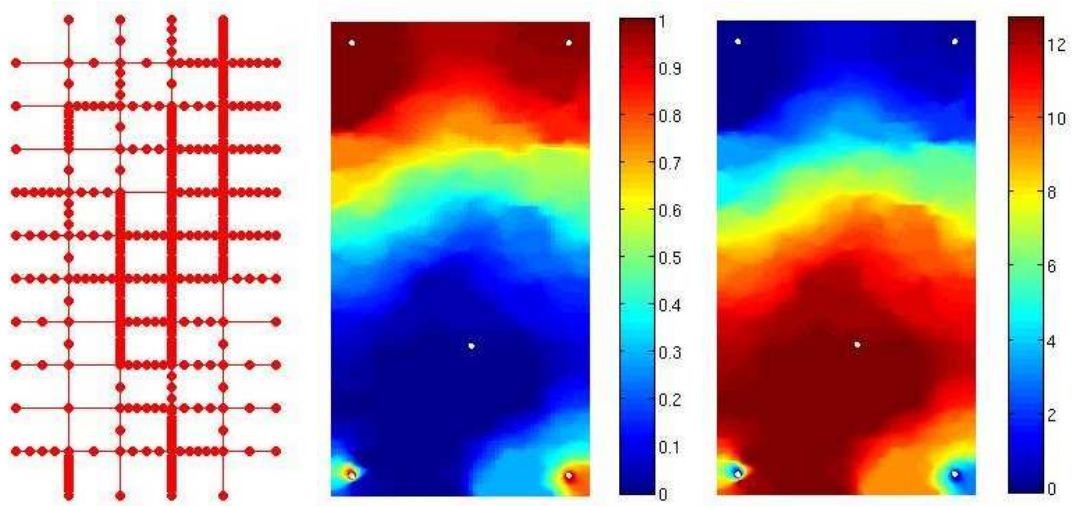

FIG. 8.7. Final adaptive mortar grid (left), final pressure field (middle), and final adjoint pressure field (right).

the production well and the upper-right injection well. This is due to the channel of high permeability running between these two wells.

For comparison, we also solve a sequence of problems using uniformly refined mortar grids. In Figure 8.8, we compare the mortar error using the uniform and adaptive approaches. Clearly, the adaptive approach reaches the chosen error tolerance using far fewer mortar degrees of freedom, and hence requiring fewer subdomain solves, 


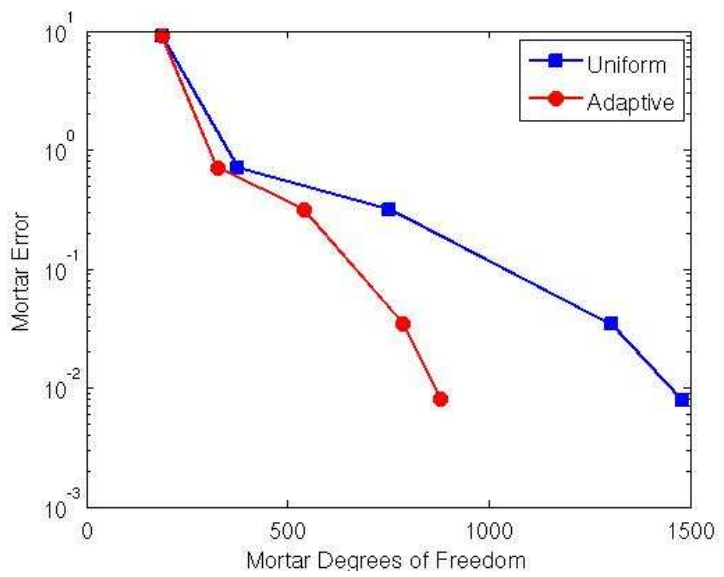

FIG. 8.8. Convergence of the mortar error under uniform and adaptive refinement of the mortars.

than the approach using uniform adaptivity.

9. Conclusions. We have shown how to derive a posteriori error estimates for linear functionals of the solution to an elliptic problem discretized using a multiscale nonoverlapping domain decomposition method. The general framework presented in section 4 allows us to consider both primal and mixed formulations of the forward and adjoint problems within each subdomain. Various subdomain discretization schemes were considered and the error estimate was shown to be accurate in all cases, even if the subdomain approximation uses an adjoint inconsistent formulation. Finally, we demonstrated that the error estimate may be decomposed into subdomain and mortar components which may be used to drive adaptive refinement.

Acknowledgments. This research was supported in part by Award No. KUK-C1013-04, made by King Abdullah University of Science and Technology (KAUST).

\section{REFERENCES}

[1] Aavatsmark, I., Eigestad, G. T., Klausen, R. A., Wheeler, M. F., and Yotov, I. Convergence of a symmetric MPFA method on quadrilateral grids. Comput. Geosci. 11, 4 (2007), $333-345$.

[2] Adams, R. A. Sobolev spaces. Academic Press, New York-London, 1975. Pure and Applied Mathematics, Vol. 65.

[3] Agélas, L., and Masson, R. Convergence of the finite volume MPFA O scheme for heterogeneous anisotropic diffusion problems on general meshes. C. R. Math. Acad. Sci. Paris 346 (2008), 1007-1012.

[4] Arbogast, T., Cowsar, L. C., Wheeler, M. F., and Yotov, I. Mixed finite element methods on nonmatching multiblock grids. SIAM J. Numer. Anal. 37, 4 (2000), 1295-1315.

[5] Arbogast, T., Dawson, C. N., Keenan, P. T., Wheeler, M. F., and Yotov, I. Enhanced cell-centered finite differences for elliptic equations on general geometry. SIAM J. Sci. Comput. 19, 2 (1998), 404-425.

[6] Arbogast, T., Pencheva, G., Wheeler, M. F., and Yotov, I. A multiscale mortar mixed finite element method. Multiscale Model. Simul. 6, 1 (2007), 319-346.

[7] Arbogast, T., Wheeler, M. F., and Yotov, I. Mixed finite elements for elliptic problems with tensor coefficients as cell-centered finite differences. SIAM J. Numer. Anal. 34, 2 (1997), 828-852.

[8] Arnold, D. N., Brezzi, F., Cockburn, B., and Marini, L. D. Unified analysis of discontinuous Galerkin methods for elliptic problems. SIAM J. Numer. Anal. 39, 5 (2001/02), 1749-1779. 
[9] Bangerth, W., And Rannacher, R. Adaptive Finite Element Methods for Differential Equations. Birkhauser Verlag, 2003.

[10] Baranger, J., Maître, J.-F., and Oudin, F. Connection between finite volume and mixed finite element methods. RAIRO Modél. Math. Anal. Numér. 30, 4 (1996), 445-465.

[11] Becker, R., And Rannacher, R. An optimal control approach to a posteriori error estimation in finite element methods. Acta Numer. 10 (2001), 1-102.

[12] Bergam, A., Bernardi, C., Hecht, F., and Mghazli, Z. Error indicators for the mortar finite element discretization of a parabolic problem. Numer. Algorithms 34, 2-4 (2003), $187-201$.

[13] Bernardi, C., And Hecht, F. Error indicators for the mortar finite element discretization of the Laplace equation. Math. Comp. 71, 240 (2002), 1371-1403.

[14] Bernardi, C., Rebollo, T. C., Hecht, F., and Mghazli, Z. Mortar finite element discretization of a model coupling Darcy and Stokes equations. M2AN Math. Model. Numer. Anal. 42, 3 (2008), 375-410.

[15] Brezzi, F., And Fortin, M. Mixed and hybrid finite element methods, vol. 15 of Springer Series in Computational Mathematics. Springer-Verlag, New York, 1991.

[16] Carey, V., Estep, D., and Tavener, S. A posteriori analysis and adaptive error control for multiscale operator decomposition solution of elliptic systems I: Triangular systems. SIAM J. Numer. Anal., 47 (2009), 740-761.

[17] Christie, M., And Blunt, M. Tenth SPE comparative solution project: a comparison of upscaling techniques. SPE Reservoir Evaluation \& Engineering 4, 4 (2001), 308-317.

[18] Ciarlet, P. G. Basic error estimates for elliptic problems. In Handbook of numerical analysis, Vol. II. North-Holland, Amsterdam, 1991, pp. 17-351.

[19] Cockburn, B., Gopalakrishnan, J., and Lazarov, R. Unified hybridization of discontinuous galerkin, mixed, and continuous galerkin methods for second order elliptic problems. SIAM J. Numer. Anal. 47, 2 (2009), 1319-1365.

[20] Eriksson, K., Estep, D., Hansbo, P., and Johnson, C. Computational Differential Equations. Cambridge University Press, 1996.

[21] Ern, A., Stephansen, A. F., And Vohralík, M. Guaranteed and robust discontinuous Galerkin a posteriori error estimates for convection-diffusion-reaction problems. J. Comput. Appl. Math. 234, 1 (2010), 114-130.

[22] ERn, A., AND Vohralík, M. Flux reconstruction and a posteriori error estimation for discontinuous Galerkin methods on general nonmatching grids. C. R. Math. Acad. Sci. Paris 347 (2009), 441-444.

[23] Estep, D. A posteriori error bounds and global error control for approximation of ordinary differential equations. SIAM J. Numer. Anal. 32, 1 (1995), 1-48.

[24] Estep, D., And French, D. Global error control for the continuous Galerkin finite element method for ordinary differential equations. RAIRO Modél. Math. Anal. Numér. 28 (1994), $815-852$.

[25] Estep, D., Ginting, V., Shadid, J., and Tavener, S. An a posteriori-a priori analysis of multiscale operator splitting. SIAM J. Numer. Anal. 46 (2008), 1116-1146.

[26] Estep, D., Larson, M. G., and Williams, R. D. Estimating the error of numerical solutions of systems of reaction-diffusion equations. Mem. Amer. Math. Soc. 146, 696 (2000), viii+109.

[27] Estep, D., Pernice, M., Pham, D., Tavener, S. J., And Wang, H. Y. A posteriori error analysis of a cell-centered finite volume method for semilinear elliptic problems. J. Comp. Appl. Math. 233 (2009), 459-472.

[28] Estep, D., Tavener, S., And Wildey, T. A posteriori analysis and improved accuracy for an operator decomposition solution of a conjugate heat transfer problem. SIAM J. Numer. Anal. 46 (2008), 2068-2089.

[29] Estep, D., Tavener, S., And Wildey, T. A posteriori error estimation and adaptive mesh refinement for a multiscale operator decomposition approach to fluid-solid heat transfer. J. Comput. Phys. 229 (June 2010), 4143-4158.

[30] Ganis, B., Pencheva, G., Wheeler, M. F., Wildey, T., and Yotov, I. A frozen Jacobian multiscale mortar preconditioner for nonlinear interface operators. Multiscale Model. Simul. 10, 3 (2012), 853-873.

[31] Ganis, B., And Yotov, I. Implementation of a mortar mixed finite element method using a multiscale flux basis. Comput. Methods Appl. Mech. Engrg. 198 (2009), 3989-3998.

[32] Giles, M. B., AND Süli, E. Adjoint methods for PDEs: a posteriori error analysis and postprocessing by duality. Acta Numer. 11 (2002), 145-236.

[33] Girault, V., Pencheva, G., Wheeler, M. F., And Wildey, T. Domain decomposition for linear elasticity with DG jumps and mortars. Comput. Methods Appl. Mech. Engrg. 198 (2009), 1751-1765. 
[34] Girault, V., Pencheva, G., Wheeler, M. F., and Wildey, T. Domain decomposition for poroelasticity and elasticity with DG jumps and mortars. M3AS 21 (2011), 169-213.

[35] Girault, V., Sun, S., Wheeler, M. F., and Yotov, I. Coupling discontinuous Galerkin and mixed finite element discretizations using mortar finite elements. SIAM J. Numer. Anal. 46, 2 (2008), 949-979.

[36] Harriman, K., Gavaghan, D., And Suli, E. The importance of adjoint consistency in the approximation of linear functionals using the discontinuous Galerkin finite element method. Technical report, Oxford University Computing Laboratory, Oxford, 2004.

[37] Hartmann, R. Adjoint consistency analysis of discontinuous Galerkin discretizations. SIAM J. Numer. Anal. 45 (October 2007), 2671-2696.

[38] Larson, M. G., and Bengzon, F. Adaptive finite element approximation of multiphysics problems. Comm. Numer. Methods Engrg. 24, 6 (2008), 505-521.

[39] Oden, J. T., and Prudhomme, S. Goal-oriented error estimation and adaptivity for the finite element method. Comput. Math. Appl. 41, 5-6 (2001), 735-756.

[40] Peaceman, D. W. Interpretation of well-block pressure in numerical reservior simulation with non-square grid blocks and anisotropic permeability. Trans. AIME 275 (1983), 10-22.

[41] Pencheva, G., Vohralik, M., Wheeler, M. F., and Wildey, T. A posteriori error control and adaptivity for multiscale, multinumerics, and mortar coupling. Accepted for publication in SIAM J. Numer. Anal., 2012.

[42] Peszyńska, M. Mortar adaptivity in mixed methods for flow in porous media. Int. J. Numer. Anal. Model. 2, 3 (2005), 241-282.

[43] Peszyńska, M., Wheeler, M., and Yotov, I. Mortar upscaling for multiphase flow in porous media. Computational Geosciences 6, 1 (2002), 73-100.

[44] Pousin, J., And Sassi, T. A posteriori error estimates and domain decomposition with nonmatching grids. Adv. Comput. Math. 23, 3 (2005), 241-263.

[45] Prager, W., And Synge, J. L. Approximations in elasticity based on the concept of function space. Quart. Appl. Math. 5 (1947), 241-269.

[46] Quarteroni, A., And Valli, A. Domain decomposition methods for partial differential equations. Numerical Mathematics and Scientific Computation. The Clarendon Press Oxford University Press, New York, 1999. Oxford Science Publications.

[47] Riviere, B. Discontinuous Galerkin Methods for Solving Elliptic and Parabolic Equations: Theory and Implementation, vol. 35 of Frontiers in Mathematics. SIAM, 2008.

[48] Rivière, B., Wheeler, M. F., And Girault, V. A priori error estimates for finite element methods based on discontinuous approximation spaces for elliptic problems. SIAM J. Numer. Anal. 39, 3 (2001), 902-931.

[49] Roberts, J. E., And Thomas, J.-M. Mixed and hybrid methods. In Handbook of Numerical Analysis, Vol. II. North-Holland, Amsterdam, 1991, pp. 523-639.

[50] Tavener, L. A. S., And Kay, D. Adjoint-based a posteriori error estimation for coupled multiphysics systems I: Steady problems. Preprint, 2012.

[51] Vohralík, M. A posteriori error estimates for lowest-order mixed finite element discretizations of convection-diffusion-reaction equations. SIAM J. Numer. Anal. 45, 4 (2007), 1570-1599.

[52] Vohralík, M. Unified primal formulation-based a priori and a posteriori error analysis of mixed finite element methods. Math. Comp. (2009). Accepted for publication.

[53] Wheeler, M., Wildey, T., And Yotov, I. A multiscale preconditioner for stochastic mortar mixed finite elements. Comput. Methods Appl. Mech. Engrg. 200, 9-12 (2011), 1251-1262.

[54] Wheeler, M., Xue, G., And Yotov, I. A multipoint flux mixed finite element method on distorted quadrilaterals and hexahedra. Numer. Math. 121, 1 (May 2012), 165-204.

[55] Wheeler, M. F., Xue, G., and Yotov, I. A multiscale mortar multipoint flux mixed finite element method. ESAIM: Math. Model. Numer. Anal. 46, 04 (2012), 759-796.

[56] Wheeler, M. F., And Yotov, I. A posteriori error estimates for the mortar mixed finite element method. SIAM J. Numer. Anal. 43, 3 (2005), 1021-1042.

[57] Wildey, T. A posteriori analysis of interior penalty discontinuous Galerkin methods. Submitted to SIAM J. Numer. Anal., 2012.

[58] Wildey, T., Estep, D., And Tavener, S. A posteriori error estimation of boundary flux. Commun. Numer. Meth. Engr. 24 (2008), 421-434.

[59] Wohlmuth, B. I. Hierarchical a posteriori error estimators for mortar finite element methods with Lagrange multipliers. SIAM J. Numer. Anal. 36, 5 (1999), 1636-1658 (electronic).

[60] Wohlmuth, B. I. A residual based error estimator for mortar finite element discretizations. Numer. Math. 84, 1 (1999), 143-171.

[61] Yотоv, I. A multilevel Newton-Krylov interface solver for multiphysics couplings of flow in porous media. Numer. Linear Algebra Appl 8, 8 (2001), 551-570.

[62] Yотоv, I. Interface solvers and preconditioners of domain decomposition type for multiphase 
flow in multiblock porous media. Nova Science Publishers, Inc., Commack, NY, USA, 2001, pp. 157-167. 



\section{RECENT REPORTS}

12/85 A two-pressure model for slightly compressible single phase flow in bi-structured porous media

Schlackow

Marguerat

Proudfoot

Bähler

Erban

Gullerova

12/86 Boolean modelling reveals new regulatory connections between

Lovrics transcription factors orchestrating the development of the ventral spinal cord

Gao

Juhász

Bock

Byrne

Dinnyés

Kovács

12/87 Asymptotic solutions of glass temperature profiles during steady optical fibre drawing

Taroni

Breward

Cummings

Griffiths

12/88 The kinetics of surfactant desorption at the airsolution interface

Morgan

Breward

Griffiths

Howell

Penfold

Thomas

Tucker

Petkov

Webster

12/89 An experimental and theoretical investigation of particlewall impacts in a T-junction

Vigolo

Griffiths

Radl

Stone

12/90 Transitions through Critical Temperatures in Nematic Liquid Crystals

Majumdar

Ockendon

Howell

Surovyatkina

12/91 Biaxial defect cores in nematic equilibria: an asymptotic result

Majumdar

Pisante

Henao

12/92 The Three Sphere Swimmer in a Nonlinear Viscoelastic Medium

Curtis

Gaffney

12/93 Diffusion of multiple species with excluded-volume effects

Bruna

Chapman

12/94 The Mechanics of a Chain or Ring of Spherical Magnets

Hall

Vella

Goriely

12/95 On-Lattice Agent-based Simulation of Populations of Cells within

Figueredo the Open-Source Chaste Framework

Joshi

Osborne

Byrne 
12/99 Calculus from the past: Multiple Delay Systems arising in Cancer Cell Modelling

12/100 Nonlocal models of electrical propagation in cardiac tissue: elec-

Bueno-Orovio trotonic effects and the modulated dispersion of repolarization

Kay

Grau

Rodriguez

Burrage

12/101 Microfluidic Immunomagnetic Multi-Target Sorting A Model for

Tsai

Controlling Deflection of Paramagnetic Beads

Griffiths

Stone

12/102 A New Lattice Boltzmann Equation to Simulate Density-Driven

Allen

Convection of Carbon Dioxide

Reis

Sun

12/103 Control and optimization of solute transport in a porous tube

Griffiths

Howell

Shipley

12/104 Air-cushioning in impact problems

Moore

Ockendon

Oliver

12/105 Strain controlled biaxial stretch: An experimental characterization

Pancheri of natural rubber

Dorfmann

12/106 Non-linear modeling of active biohybrid materials

Paetsch

Dorfmann

12/107 Coalescence of Liquid Drops: Different Models Versus Experi-

Sprittles ment

Shikhmurzaev

Copies of these, and any other OCCAM reports can be obtained from:

Oxford Centre for Collaborative Applied Mathematics

Mathematical Institute

24 - 29 St Giles'

Oxford

OX1 3LB

England

www.maths.ox.ac.uk/occam 